\title{
Pacific
}

Journal of

Mathematics

\section{CHARACTER ANALOGUES OF RAMANUJAN-TYPE} INTEGRALS INVOLVING THE RIEMANN $\Xi$-FUNCTION

\author{
ATUl DiXIT
}




\title{
CHARACTER ANALOGUES OF RAMANUJAN-TYPE INTEGRALS INVOLVING THE RIEMANN $\Xi$-FUNCTION
}

\author{
ATUl DiXIT
}

\begin{abstract}
A new class of integrals involving the product of $\Xi$-functions associated with primitive Dirichlet characters is considered. These integrals give rise to transformation formulas of the type

$$
F(z, \alpha, \chi)=F(-z, \beta, \bar{\chi})=F(-z, \alpha, \bar{\chi})=F(z, \beta, \chi),
$$

where $\alpha \beta=1$. New character analogues of the Ramanujan-Guinand formula, the Koshliakov's formula, and a transformation formula of Ramanujan, as well as its recent generalization, are shown as particular examples. Finally, character analogues of a conjecture of Ramanujan, and Hardy and Littlewood involving infinite series of Möbius functions are derived.
\end{abstract}

\section{Introduction}

Modular transformations are ubiquitous in Ramanujan's notebooks [1957] and in his "Lost Notebook" [1988]. Ramanujan usually expressed them in a symmetric way, and they were valid under the conditions $\alpha \beta=\pi$, or $\alpha \beta=\pi^{2}, \ldots$ In the same spirit, on page 220 in one of the manuscripts of Ramanujan in the handwriting of Watson [Ramanujan 1988], one finds the following beautiful claim.

Theorem 1.1. Define

$$
\lambda(x):=\psi(x)+\frac{1}{2 x}-\log x,
$$

where

$$
\psi(x):=\frac{\Gamma^{\prime}(x)}{\Gamma(x)}=-\gamma-\sum_{m=0}^{\infty}\left(\frac{1}{m+x}-\frac{1}{m+1}\right)
$$

is the logarithmic derivative of the Gamma function. Let the Riemann $\xi$-function be defined by

$$
\xi(s):=(s-1) \pi^{-s / 2} \Gamma\left(1+\frac{1}{2} s\right) \zeta(s),
$$

2010 Mathematics Subject Classification.

MSC2010: primary 11M06; secondary 11M35.

Keywords: Dirichlet character, Dirichlet L-function, modified Bessel function, Möbius function,

Mellin transform, Ramanujan, Hardy, Littlewood, Koshliakov, Guinand. 
and let

$$
\Xi(t):=\xi\left(\frac{1}{2}+i t\right)
$$

be the Riemann $\Xi$-function. If $\alpha$ and $\beta$ are positive numbers such that $\alpha \beta=1$, then

$$
\begin{array}{r}
\sqrt{\alpha}\left(\frac{\gamma-\log (2 \pi \alpha)}{2 \alpha}+\sum_{k=1}^{\infty} \lambda(k \alpha)\right)=\sqrt{\beta}\left(\frac{\gamma-\log (2 \pi \beta)}{2 \beta}+\sum_{k=1}^{\infty} \lambda(k \beta)\right) \\
=-\frac{1}{\pi^{3 / 2}} \int_{0}^{\infty}\left|\Xi\left(\frac{1}{2} t\right) \Gamma\left(\frac{-1+i t}{4}\right)\right|^{2} \frac{\cos ((t / 2) \log \alpha)}{1+t^{2}} d t,
\end{array}
$$

where $\gamma$ denotes Euler's constant.

This identity is of a special kind since it contains not only a modular transformation, but also a beautiful integral involving the Riemann $\Xi$-function. In fact, the invariance of the integral in (1-1) under the map $\alpha \rightarrow \beta$ establishes the equality of the first and the second expressions in (1-1). This is used in [Berndt and Dixit 2010 ] to prove the claim above and in [Dixit 2010; 2011a; 2011b] to obtain many transformation formulas of the type $F(\alpha)=F(\beta)$ or $F(z, \alpha)=F(z, \beta)$, where $\alpha \beta=1$ and an integral involving the Riemann $\Xi$-function is always linked to them. This gives new identities involving infinite series of the Hurwitz zeta function as well as extensions of some well-known formulas like the Ramanujan-Guinand formula, discovered first by Ramanujan [1988, p. 253] and later in a different but equivalent form by Guinand [1955], and a formula of Koshliakov [1928], also in the lost notebook [Ramanujan 1988, p. 254]; see [Berndt et al. 2008; Dixit 2011b]. For example, we mention the following generalization of Theorem 1.1:

Theorem 1.2 [Dixit 2011a; 2011b]. Let $-1<\operatorname{Re} z<1$. Define $\varphi(z, x)$ by

$$
\varphi(z, x)=\zeta(z+1, x)-\frac{x^{-z}}{z}-\frac{1}{2} x^{-z-1},
$$

where $\zeta(z, x)$ denotes the Hurwitz zeta function. If $\alpha$ and $\beta$ are any positive numbers such that $\alpha \beta=1$, then

$$
\begin{aligned}
& \alpha^{\frac{z+1}{2}}\left(\sum_{n=1}^{\infty} \varphi(z, n \alpha)-\frac{\zeta(z+1)}{2 \alpha^{z+1}}-\frac{\zeta(z)}{\alpha z}\right) \\
&=\beta^{(z+1) / 2}\left(\sum_{n=1}^{\infty} \varphi(z, n \beta)-\frac{\zeta(z+1)}{2 \beta^{z+1}}-\frac{\zeta(z)}{\beta z}\right) \\
&=\frac{8(4 \pi)^{(z-3) / 2}}{\Gamma(z+1)} \int_{0}^{\infty} \Gamma\left(\frac{z-1+i t}{4}\right) \Gamma\left(\frac{z-1-i t}{4}\right) \\
& \times \Xi\left(\frac{t+i z}{2}\right) \Xi\left(\frac{t-i z}{2}\right) \frac{\cos ((t / 2) \log \alpha)}{(z+1)^{2}+t^{2}} d t
\end{aligned}
$$

where $\Xi(t)$ is the Riemann $\Xi$-function. 
Another example of a transformation formula of the type $F(z, \alpha)=F(z, \beta)$ along with an integral involving Riemann's $\Xi$-functions is the following extended version of the Ramanujan-Guinand formula just mentioned:

Theorem 1.3 [Dixit 2011b, Theorem 1.4]. Let $K_{v}(s)$ denote the modified Bessel function of order $v$, let $\gamma$ denote Euler's constant and let $\sigma_{k}(n)=\sum_{d \mid n} d^{k}$. Let $-1<\operatorname{Re} z<1$. Then if $\alpha$ and $\beta$ are positive numbers such that $\alpha \beta=1$, we have

$$
\begin{aligned}
& \sqrt{\alpha}\left(\alpha^{z / 2-1} \pi^{-z / 2} \Gamma\left(\frac{z}{2}\right) \zeta(z)\right. \\
& \left.\quad+\alpha^{-z / 2-1} \pi^{z / 2} \Gamma\left(\frac{-z}{2}\right) \zeta(-z)-4 \sum_{n=1}^{\infty} \sigma_{-z}(n) n^{z / 2} K_{z / 2}(2 n \pi \alpha)\right) \\
& =\sqrt{\beta}\left(\beta^{z / 2-1} \pi^{-z / 2} \Gamma\left(\frac{z}{2}\right) \zeta(z)\right. \\
& \left.\quad+\beta^{-z / 2-1} \pi^{z / 2} \Gamma\left(\frac{-z}{2}\right) \zeta(-z)-4 \sum_{n=1}^{\infty} \sigma_{-z}(n) n^{z / 2} K_{z / 2}(2 n \pi \beta)\right) \\
& =-\frac{32}{\pi} \int_{0}^{\infty} \Xi\left(\frac{t+i z}{2}\right) \Xi\left(\frac{t-i z}{2}\right) \frac{\cos ((t / 2) \log \alpha)}{\left(t^{2}+(z+1)^{2}\right)\left(t^{2}+(z-1)^{2}\right)} d t
\end{aligned}
$$

Letting $z \rightarrow 0$ in (1-3) then gives an extended version of Koshliakov's formula: Theorem 1.4 [Dixit 2010]. Let $d(n)$ denote the number of positive divisors of $n$, and let $K_{0}(n)$ denote the modified Bessel function of order 0 . If $\alpha$ and $\beta$ are positive numbers such that $\alpha \beta=1$, then

$$
\begin{aligned}
\sqrt{\alpha}\left(\frac{\gamma-\log (4 \pi \alpha)}{\alpha}-4 \sum_{n=1}^{\infty} d(n)\right. & \left.K_{0}(2 \pi n \alpha)\right) \\
& =\sqrt{\beta}\left(\frac{\gamma-\log (4 \pi \beta)}{\beta}-4 \sum_{n=1}^{\infty} d(n) K_{0}(2 \pi n \beta)\right) \\
& =-\frac{32}{\pi} \int_{0}^{\infty} \frac{(\Xi(t / 2))^{2} \cos \left(\frac{1}{2} t \log \alpha\right) d t}{\left(1+t^{2}\right)^{2}} .
\end{aligned}
$$

By an "extended version", we mean that the original identity known before is linked to an integral involving the Riemann $\Xi$-function.

N. S. Koshliakov [1934a; 1934b; 1936; 1949; 1954] ${ }^{1}$ was another mathematician who did significant research in this area after Ramanujan. Besides using contour integration, Mellin transforms, and several summation formulas that he developed, he frequently used a method similar to that developed by Ramanujan [1915; 1927, pp. 72-77] to obtain old and new transformation formulas of the form

\footnotetext{
${ }^{1}$ For the genesis of the monograph [Koshlyakov 1949], written under the patronymic "N. S. Sergeev", see [Bogolyubov et al. 1990, pp. 198-199].
} 
$F(\alpha)=F(\beta)$, where $\alpha \beta=k$ for some constant $k$. He obtained deep generalizations of well-known formulas of Ramanujan and of Hardy (such as [Hardy 1915, (2)]), some of them being analogues in rational and number fields. Koshliakov [1934c; 1937] also used Fourier's integral theorem to obtain expressions for the Riemann $\Xi$-function, a method also enunciated in [Ramanujan 1915]. Around the same time, Ferrar [1936] also worked on transformation formulas of this kind.

As can be seen from (1-1), the general form of the integrals giving rise to formulas of the type $F(\alpha)=F(\beta)$, where $\alpha \beta=1$, is

$$
\int_{0}^{\infty} f\left(\frac{t}{2}\right) \Xi\left(\frac{t}{2}\right) \cos \mu t d t
$$

for $\mu$ real and $f(t)=\phi(i t) \phi(-i t)$, where $\phi$ is analytic in $t$ as a function of a real variable. This integral is mentioned in [Titchmarsh 1986, p. 35]. Similarly, from (1-2) and (1-3), it is clear that the general form of the integrals giving rise to identities of the type $F(z, \alpha)=F(z, \beta)$, where $\alpha \beta=1$, is

$$
\int_{0}^{\infty} f\left(z, \frac{t}{2}\right) \Xi\left(\frac{t+i z}{2}\right) \Xi\left(\frac{t-i z}{2}\right) \cos \mu t d t,
$$

for $\mu$ real and $f(z, t)=\phi(z, i t) \phi(z,-i t)$, where $\phi$ is both analytic in $t$ as a function of a real variable and analytic in $z$ in some complex domain. An integral of this kind was first introduced by Ramanujan [1915].

In this article, we find character analogues of all of the above-mentioned theorems. The character analogue of the Ramanujan-Guinand formula, and hence of Koshliakov's formula, given here differs from the ones established in [Berndt et al. 2011]. Throughout this article, we will be concerned with the principal branch of the logarithm. Since we frequently use the functional equation for $L$-functions (see (1-10) below), we work only with a primitive, nonprincipal Dirichlet character $\chi$ modulo $q$, where $q$ is the period of the character; see [Apostol 1972, Theorem 1]. It is easy to see that its conjugate character $\bar{\chi}$ is also a primitive, nonprincipal character modulo $q$ and $\bar{\chi}$ is even (odd) if and only if $\chi$ is even (respectively odd). Let $L(s, \chi)$ denote the Dirichlet $L$-function defined by $L(s, \chi)=\sum_{n=1}^{\infty} \chi(n) / n^{s}$ for $\operatorname{Re} s>1$. This series converges conditionally for $0<\operatorname{Re} s<1$. Also, it can be analytically continued to an entire function of $s$. Let $G(\chi):=G(1, \chi)$, where $G(n, \chi)$ is the Gauss sum defined by

$$
G(n, \chi):=\sum_{m=1}^{q} \chi(m) e^{2 \pi i m n / q} .
$$

We know that [Apostol 1976, p. 168]

$$
|G(\chi)|^{2}=q,
$$


and it is easy to see that

$$
\overline{G(\chi)}= \begin{cases}G(\bar{\chi}) & \text { for } \chi \text { even } \\ -G(\bar{\chi}) & \text { for } \chi \text { odd. }\end{cases}
$$

Define $b$ as follows:

$$
b= \begin{cases}0 & \text { for } \chi(-1)=1 \\ 1 & \text { for } \chi(-1)=-1\end{cases}
$$

Then the function $\xi(s, \chi)$ is defined by

$$
\xi(s, \chi):=\left(\frac{\pi}{q}\right)^{-(s+b) / 2} \Gamma\left(\frac{s+b}{2}\right) L(s, \chi),
$$

and the analogue of the Riemann $\Xi$-function for Dirichlet characters is defined as

$$
\Xi(t, \chi):=\xi\left(\frac{1}{2}+i t, \chi\right) \text {. }
$$

$L$-functions satisfy the functional equation [Apostol 1976, p. 263]

$$
L(1-s, \chi)=\frac{q^{s-1} \Gamma(s)}{(2 \pi)^{s}}\left(e^{-\pi i s / 2}+\chi(-1) e^{\pi i s / 2}\right) G(\chi) L(s, \bar{\chi}),
$$

which can be rephrased in terms of $\xi(s, \chi)$ as [Davenport 2000]

$$
\xi(1-s, \bar{\chi})=\epsilon(\chi) \xi(s, \chi),
$$

where $\epsilon(\chi)=i^{b} q^{1 / 2} / G(\chi)$. By (1-5), $|\epsilon(\chi)|=1$. Next, we note Stirling's formula in a vertical strip $\alpha \leq \sigma \leq \beta, s=\sigma+i$, namely,

$$
|\Gamma(s)|=(2 \pi)^{\frac{1}{2}}|t|^{\sigma-\frac{1}{2}} e^{-\frac{1}{2} \pi|t|}\left(1+O\left(\frac{1}{|t|}\right)\right)
$$

uniformly as $|t| \rightarrow \infty$. Now, using (1-10) and the fact that $|L(s, \chi)|=O(q|t|)$ for $\operatorname{Re} s \geq \frac{1}{2}$ [Davenport 2000, p. 82], we easily see that for $\operatorname{Re} s \geq-\delta, \delta>0$, we have

$$
L(s, \chi)=O\left(q^{\frac{3}{2}+\delta}|t|^{\frac{3}{2}+\delta}\right) .
$$

We will subsequently use this result.

Transformation formulas involving Dirichlet characters of the form

where

$$
\sum_{n=1}^{\infty} \chi(n) f(n)=\sum_{n=1}^{\infty} \bar{\chi}(n) g(n)
$$

$$
g(x)= \begin{cases}\frac{2 G(\chi)}{q} \int_{0}^{\rightarrow \infty} \cos \left(\frac{2 \pi x t}{q}\right) f(t) d t & \text { for } \chi(-1)=1, \\ \frac{-2 i G(\chi)}{q} \int_{0}^{\rightarrow \infty} \sin \left(\frac{2 \pi x t}{q}\right) f(t) d t & \text { for } \chi(-1)=-1,\end{cases}
$$


were considered by Guinand [1941, Theorems 4 and 5], though he did not give particular examples. Here, we derive a character analogue of the integral in (1-4). Its general form is

$$
\int_{0}^{\infty} f\left(z, \frac{t}{2}\right) \Xi\left(\frac{t+i z}{2}, \bar{\chi}\right) \Xi\left(\frac{t-i z}{2}, \chi\right) \cos \left(\frac{1}{2} t \log \alpha\right) d t,
$$

where $f$ is an even function of both the variables $z$ and $t$. These integrals give rise to transformation formulas of the type $F(z, \alpha, \chi)=F(-z, \beta, \bar{\chi})=F(-z, \alpha, \bar{\chi})=$ $F(z, \beta, \chi)$. Then, via Fourier's integral theorem, one may be able to obtain integral representations for $\Xi((t+i z) / 2, \bar{\chi}) \Xi((t-i z) / 2, \chi)$, which are of independent interest. The character analogue of Theorem 1.3 is as follows.

Theorem 1.5. Let $-1<\operatorname{Re} z<1$ and let $\chi$ denote a primitive, nonprincipal character modulo $q$. Let the number $b$ be defined as in (1-7). Let $K_{v}(z), d(n)$, and $\gamma$ be defined as before, and let $\alpha$ and $\beta$ be positive numbers such that $\alpha \beta=1$. If

$$
F(z, \alpha, \chi):=\alpha^{b+\frac{1}{2}} \sum_{n=1}^{\infty} \chi(n) n^{-z / 2+b}\left(\sum_{d \mid n} \bar{\chi}^{2}(d) d^{z}\right) K_{-z / 2}\left(\frac{2 \pi n \alpha}{q}\right),
$$

then

$$
\begin{aligned}
F(z, \alpha, \chi) & =F(-z, \beta, \bar{\chi})=F(-z, \alpha, \bar{\chi})=F(z, \beta, \chi) \\
& =\frac{1}{8 \pi} \int_{0}^{\infty} \Xi\left(\frac{t+i z}{2}, \bar{\chi}\right) \Xi\left(\frac{t-i z}{2}, \chi\right) \cos \left(\frac{1}{2} t \log \alpha\right) d t .
\end{aligned}
$$

Define $\psi(a, \chi)$ by

$$
\psi(a, \chi)=-\sum_{n=1}^{\infty} \frac{\chi(n)}{n+a},
$$

where $a \in \mathbb{C} \backslash \mathbb{Z}_{<0}$. For a real character $\chi$, this agrees with the character analogue of the psi function obtained by the logarithmic differentiation of the following Weierstrass product form of the character analogue of the gamma function for real characters derived by Berndt [1975]:

$$
\Gamma(a, \chi)=e^{-a L(1, \chi)} \prod_{n=1}^{\infty}\left(1+\frac{a}{n}\right)^{-\chi(n)} e^{a \chi(n) / n} .
$$

The character analogue of the Hurwitz zeta function $\zeta(z, a)$ is given by [Berndt 1970, Example 3.2]

$$
L(z, a, \chi)=\sum_{n=1}^{\infty} \frac{\chi(n)}{(n+a)^{z}}
$$


valid for $\operatorname{Re} z>0$, and provided $a \in \mathbb{C} \backslash \mathbb{Z}_{<0}$. The above character analogue of the Hurwitz zeta function can also be obtained as the special case when $x=0$ of the function $L(z, x, a, \chi)$ defined by [Berndt 1975]

$$
L(z, x, a, \chi):=\sum_{n=0}^{\infty} e^{2 \pi i n x / q} \chi(n)(n+a)^{-z},
$$

where the prime indicates that the term corresponding to $n=-a$ is omitted if $a$ is a negative integer and $\chi(a) \neq 0$. As shown in [Berndt 1975], $L(z, x, a, \chi)$ converges for $\operatorname{Re} z>0$ if $x$ is not an integer, or if $x$ is an integer and $\operatorname{gcd}(x, q)>1$. If $x$ is an integer and $\operatorname{gcd}(x, q)=1$, the series converges for $\operatorname{Re} z>1$. For mean value properties of $L(z, a, \chi)$ and asymptotic formulas, see [Ma et al. 2010]. The character analogues of Theorem 1.2 are given below.

Theorem 1.6. Let $\chi$ denote an even, primitive, nonprincipal character modulo $q$. Let $-1<\operatorname{Re} z<1$, and let $L(z, a, \chi)$ be defined as in (1-17). Define $T(z, \alpha, \chi)$ by

$$
T(z, \alpha, \chi):=\frac{\alpha^{z / 2} q^{z / 2} \Gamma(z+1)}{2^{z} \pi^{z / 2} G(\chi)}
$$

and $\Omega(z, t)$ by

$$
\begin{aligned}
\Omega(z, t):=\left((z+1)^{2}+t^{2}\right) \Gamma( & \left.\frac{-z-1+i t}{4}\right) \Gamma\left(\frac{-z-1-i t}{4}\right) \\
& +\left((z-1)^{2}+t^{2}\right) \Gamma\left(\frac{z-1+i t}{4}\right) \Gamma\left(\frac{z-1-i t}{4}\right) .
\end{aligned}
$$

If $\alpha$ and $\beta$ are positive numbers such that $\alpha \beta=1$, then

$$
\begin{aligned}
& \sqrt{\alpha}\left(T(z, \alpha, \chi) \sum_{n=1}^{\infty} \chi(n) L(z+1, n \alpha, \chi)\right.\left.+T(-z, \alpha, \bar{\chi}) \sum_{n=1}^{\infty} \bar{\chi}(n) L(-z+1, n \alpha, \bar{\chi})\right) \\
&+\sqrt{\beta}\left(T(-z, \beta, \bar{\chi}) \sum_{n=1}^{\infty} \bar{\chi}(n) L(-z+1, n \beta, \bar{\chi})\right. \\
&\left.+T(z, \beta, \chi) \sum_{n=1}^{\infty} \chi(n) L(z+1, n \beta, \chi)\right) \\
&=\frac{1}{64 \pi^{3 / 2} q} \int_{0}^{\infty} \Omega(z, t) \Xi\left(\frac{t+i z}{2}, \bar{\chi}\right) \Xi\left(\frac{t-i z}{2}, \chi\right) \cos \left(\frac{1}{2} t \log \alpha\right) d t .
\end{aligned}
$$

Theorem 1.7. Let $\chi$ denote an odd, primitive, nonprincipal character modulo $q$. Let $-1<\operatorname{Re} z<1$, let $L(z, a, \chi)$ be defined as in (1-17), and let $T(z, \alpha, \chi)$ be defined as in (1-18). Define $\Lambda(z, t)$ by

$$
\Lambda(z, t):=\Gamma\left(\frac{z+1+i t}{4}\right) \Gamma\left(\frac{z+1-i t}{4}\right)+\Gamma\left(\frac{-z+1+i t}{4}\right) \Gamma\left(\frac{-z+1-i t}{4}\right) .
$$


If $\alpha$ and $\beta$ are positive numbers such that $\alpha \beta=1$, then

$$
\begin{aligned}
& \sqrt{\alpha}\left(T(z, \alpha, \chi) \sum_{n=1}^{\infty} \chi(n) L(z+1, n \alpha, \chi)\right. \\
&\left.+T(-z, \alpha, \bar{\chi}) \sum_{n=1}^{\infty} \bar{\chi}(n) L(-z+1, n \alpha, \bar{\chi})\right) \\
&=\sqrt{\beta}\left(T(-z, \beta, \bar{\chi}) \sum_{n=1}^{\infty} \bar{\chi}(n) L(-z+1, n \beta, \bar{\chi})\right.\left.+T(z, \beta, \chi) \sum_{n=1}^{\infty} \chi(n) L(z+1, n \beta, \chi)\right) \\
&=\frac{1}{4 \pi^{1 / 2} i q^{2}} \int_{0}^{\infty} \Lambda(z, t) \Xi\left(\frac{t+i z}{2}, \bar{\chi}\right) \Xi\left(\frac{t-i z}{2}, \chi\right) \cos \left(\frac{1}{2} t \log \alpha\right) d t
\end{aligned}
$$

The following interesting identity was suggested by the work of Ramanujan:

Theorem 1.8 ([Hardy and Littlewood 1916, p. 156, Section 2.5]). Let $\mu(n)$ denote the Möbius function. Let $\alpha$ and $\beta$ be two positive numbers such that $\alpha \beta=1$. Assume that the series

$$
\sum_{\rho} \frac{\Gamma((1-\rho) / 2)}{\zeta^{\prime}(\rho)} a^{\rho}
$$

converges, where $\rho$ runs through the nontrivial zeros of $\zeta(s)$ and a denotes a positive real number, and that the nontrivial zeros of $\zeta(s)$ are simple. Then

$$
\begin{aligned}
& \sqrt{\alpha} \sum_{n=1}^{\infty} \frac{\mu(n)}{n} e^{-\pi \alpha^{2} / n^{2}}-\frac{1}{4 \sqrt{\pi} \sqrt{\alpha}} \sum_{\rho} \frac{\Gamma((1-\rho) / 2)}{\zeta^{\prime}(\rho)} \pi^{\rho / 2} \alpha^{\rho} \\
& \quad=\sqrt{\beta} \sum_{n=1}^{\infty} \frac{\mu(n)}{n} e^{-\pi \beta^{2} / n^{2}}-\frac{1}{4 \sqrt{\pi} \sqrt{\beta}} \sum_{\rho} \frac{\Gamma((1-\rho) / 2)}{\zeta^{\prime}(\rho)} \pi^{\rho / 2} \beta^{\rho} .
\end{aligned}
$$

Hardy and Littlewood's original formulation was slightly different from (1-23) but is readily seen to be equivalent to it. See also [Berndt 1998, p. 470; Paris and Kaminski 2001, p. 143; Titchmarsh 1986, p. 219, Section 9.8] for discussions on this identity. Based on certain assumptions, the character analogues of (1-23) for even and odd primitive Dirichlet characters, which furnish two examples of transformation formulas of the form $F(\alpha, \chi)=F(\beta, \bar{\chi})$, are derived here and are as follows.

Theorem 1.9. Let $\chi$ be an odd, primitive character modulo $q$, and let $\alpha$ and $\beta$ be two positive numbers such that $\alpha \beta=1$. Assume that the series

$$
\sum_{\rho} \frac{\pi^{\rho / 2} \alpha^{\rho} \Gamma((2-\rho) / 2)}{q^{\rho / 2} L^{\prime}(\rho, \chi)} \text { and } \sum_{\rho} \frac{\pi^{\rho / 2} \beta^{\rho} \Gamma((2-\rho) / 2)}{q^{\rho / 2} L^{\prime}(\rho, \bar{\chi})}
$$


converge, where $\rho$ runs through the nontrivial zeros of $L(s, \chi)$ and $L(s, \bar{\chi})$ respectively, and that the nontrivial zeros of the associated Dirichlet $L$-functions are simple. Then

$$
\begin{aligned}
& \alpha \sqrt{\alpha} \sqrt{G(\chi)}\left(\sum_{n=1}^{\infty} \frac{\chi(n) \mu(n)}{n^{2}} e^{-\pi \alpha^{2} /\left(q n^{2}\right)}-\frac{q}{4 \pi \alpha^{2}} \sum_{\rho} \frac{\Gamma\left(\frac{2-\rho}{2}\right)}{L^{\prime}(\rho, \chi)}\left(\frac{\pi}{q}\right)^{\rho / 2} \alpha^{\rho}\right) \\
= & \beta \sqrt{\beta} \sqrt{G(\bar{\chi})}\left(\sum_{n=1}^{\infty} \frac{\bar{\chi}(n) \mu(n)}{n^{2}} e^{-\pi \beta^{2} /\left(q n^{2}\right)}-\frac{q}{4 \pi \beta^{2}} \sum_{\rho} \frac{\Gamma\left(\frac{2-\rho}{2}\right)}{L^{\prime}(\rho, \bar{\chi})}\left(\frac{\pi}{q}\right)^{\rho / 2} \beta^{\rho}\right) .
\end{aligned}
$$

Theorem 1.10. Let $\chi$ be an even, primitive character modulo $q$, and let $\alpha$ and $\beta$ be two positive numbers such that $\alpha \beta=1$. Assume that the series

$$
\sum_{\rho} \frac{\pi^{\rho / 2} \alpha^{\rho} \Gamma((2-\rho) / 2)}{q^{\rho / 2} L^{\prime}(\rho, \chi)} \text { and } \sum_{\rho} \frac{\pi^{\rho / 2} \beta^{\rho} \Gamma((2-\rho) / 2)}{q^{\rho / 2} L^{\prime}(\rho, \bar{\chi})}
$$

converge, where $\rho$ runs through the nontrivial zeros of $L(s, \chi)$ and $L(s, \bar{\chi})$ respectively, and that the nontrivial zeros of the associated Dirichlet $L$-functions are simple. Then

$$
\begin{aligned}
& \text { 25) } \sqrt{\alpha} \sqrt{G(\chi)}\left(\sum_{n=1}^{\infty} \frac{\chi(n) \mu(n)}{n} e^{-\pi \alpha^{2} /\left(q n^{2}\right)}-\frac{\sqrt{q}}{4 \sqrt{\pi} \alpha} \sum_{\rho} \frac{\Gamma\left(\frac{1-\rho}{2}\right)}{L^{\prime}(\rho, \chi)}\left(\frac{\pi}{q}\right)^{\rho / 2} \alpha^{\rho}\right) \\
& =\sqrt{\beta} \sqrt{G(\bar{\chi})}\left(\sum_{n=1}^{\infty} \frac{\bar{\chi}(n) \mu(n)}{n} e^{-\pi \beta^{2} /\left(q n^{2}\right)}-\frac{\sqrt{q}}{4 \sqrt{\pi} \beta} \sum_{\rho} \frac{\Gamma\left(\frac{1-\rho}{2}\right)}{L^{\prime}(\rho, \bar{\chi})}\left(\frac{\pi}{q}\right)^{\rho / 2} \beta^{\rho}\right) .
\end{aligned}
$$

This paper is organized as follows. In Section 2, we give a complex integral representation of (1-14) that is used in subsequent sections. In Section 3, we prove Theorem 1.5. Then in Section 4, we compute the inverse Mellin transforms and asymptotic expansions of certain functions which are subsequently used in Section 5. Section 5 is devoted to proofs of Theorems 1.6 and 1.7. Character analogues of Ramanujan's transformation formula (Theorem 1.1) are derived as special cases of these theorems. We conclude this section with a curious result on a certain double series involving characters. In Section 6, we present proofs of Theorems 1.9 and 1.10. Finally we conclude with some open problems in Section 7.

\section{A complex integral representation of (1-14)}

In this section, we give a formal way of transforming an integral involving a character analogue of Riemann's $\Xi$-function into an equivalent complex integral which allows us to use residue calculus and Mellin transform techniques for its evaluation. 
Theorem 2.1. Let

$$
f(z, t)=\frac{\phi(z, i t) \phi(z,-i t)+\phi(-z, i t) \phi(-z,-i t)}{2},
$$

where $\phi$ is analytic in $t$ as a function of a real variable and analytic in $z$ in some complex domain. Let $y=e^{\mu}$ with $\mu$ real. Then, under the assumption that the integral on the left side below converges,

$$
\begin{aligned}
& \int_{0}^{\infty} f(z, t) \Xi\left(t+\frac{i z}{2}, \bar{\chi}\right) \Xi\left(t-\frac{i z}{2}, \chi\right) \cos \mu t d t \\
& =\frac{1}{4 i \sqrt{y}} \int_{\frac{1}{2}-i \infty}^{\frac{1}{2}+i \infty}\left(\phi\left(z, s-\frac{1}{2}\right) \phi\left(z, \frac{1}{2}-s\right)+\phi\left(-z, s-\frac{1}{2}\right) \phi\left(-z, \frac{1}{2}-s\right)\right) \\
& \times \xi\left(s-\frac{z}{2}, \bar{\chi}\right) \xi\left(s+\frac{z}{2}, \chi\right) y^{s} d s .
\end{aligned}
$$

Proof. Let

$$
I(z, \mu, \chi):=\int_{0}^{\infty} f(z, t) \Xi\left(t+\frac{i z}{2}, \bar{\chi}\right) \Xi\left(t-\frac{i z}{2}, \chi\right) \cos \mu t d t .
$$

Then

$$
\begin{array}{r}
I(z, \mu, \chi)=\frac{1}{2}\left(\int_{0}^{\infty} f(z, t) \Xi\left(t+\frac{i z}{2}, \bar{\chi}\right) \Xi\left(t-\frac{i z}{2}, \chi\right) y^{i t} d t\right. \\
\left.\quad+\int_{0}^{\infty} f(z, t) \Xi\left(t+\frac{i z}{2}, \bar{\chi}\right) \Xi\left(t-\frac{i z}{2}, \chi\right) y^{-i t} d t\right) \\
=\frac{1}{2}\left(\int_{0}^{\infty} f(z, t) \Xi\left(t+\frac{i z}{2}, \bar{\chi}\right) \Xi\left(t-\frac{i z}{2}, \chi\right) y^{i t} d t\right. \\
\left.\quad+\int_{-\infty}^{0} f(z,-t) \Xi\left(-t+\frac{i z}{2}, \bar{\chi}\right) \Xi\left(-t-\frac{i z}{2}, \chi\right) y^{i t} d t\right) .
\end{array}
$$

However, using (1-11), we readily see that

$$
\begin{aligned}
\Xi\left(-t+\frac{i z}{2}, \bar{\chi}\right) & =\xi\left(\frac{1}{2}-i t-\frac{z}{2}, \bar{\chi}\right) \\
& =\epsilon(\chi) \xi\left(\frac{1}{2}+i t+\frac{z}{2}, \chi\right)=\epsilon(\chi) \Xi\left(t-\frac{i z}{2}, \chi\right) \\
\Xi\left(-t-\frac{i z}{2}, \chi\right) & =\xi\left(\frac{1}{2}-i t+\frac{z}{2}, \chi\right) \\
& =(\epsilon(\chi))^{-1} \xi\left(\frac{1}{2}+i t-\frac{z}{2}, \bar{\chi}\right)=(\epsilon(\chi))^{-1} \Xi\left(t+\frac{i z}{2}, \bar{\chi}\right)
\end{aligned}
$$

so that

$$
\Xi\left(-t+\frac{i z}{2}, \bar{\chi}\right) \Xi\left(-t-\frac{i z}{2}, \chi\right)=\Xi\left(t+\frac{i z}{2}, \bar{\chi}\right) \Xi\left(t-\frac{i z}{2}, \chi\right)
$$


Thus from (2-3), (2-4), and the fact that $f$ is an even function of $t$, we obtain

$$
\begin{aligned}
& I(z, \mu, \chi) \\
& =\frac{1}{2} \int_{-\infty}^{\infty} f(z, t) \Xi\left(t+\frac{i z}{2}, \bar{\chi}\right) \Xi\left(t-\frac{i z}{2}, \chi\right) y^{i t} d t \\
& =\frac{1}{4 i \sqrt{y}} \int_{\frac{1}{2}-i \infty}^{\frac{1}{2}+i \infty}\left(\phi\left(z, s-\frac{1}{2}\right) \phi\left(z, \frac{1}{2}-s\right)+\phi\left(-z, s-\frac{1}{2}\right) \phi\left(-z, \frac{1}{2}-s\right)\right) \\
& \times \xi\left(s-\frac{z}{2}, \bar{\chi}\right) \xi\left(s+\frac{z}{2}, \chi\right) y^{s} d s,
\end{aligned}
$$

where in the penultimate line, we made the change of variable $s=\frac{1}{2}+i t$.

For our purpose here, we replace $\mu$ by $2 \mu$ in (2-2) and then $t$ by $t / 2$ on the left-hand side of (2-2). Thus, with $y=e^{2 \mu}$, we find that

$$
\begin{aligned}
& \int_{0}^{\infty} f\left(z, \frac{t}{2}\right) \Xi\left(\frac{t+i z}{2}, \bar{\chi}\right) \Xi\left(\frac{t-i z}{2}, \chi\right) \cos \mu t d t \\
& =\frac{1}{2 i \sqrt{y}} \int_{\frac{1}{2}-i \infty}^{\frac{1}{2}+i \infty}\left(\phi\left(z, s-\frac{1}{2}\right) \phi\left(z, \frac{1}{2}-s\right)+\phi\left(-z, s-\frac{1}{2}\right) \phi\left(-z, \frac{1}{2}-s\right)\right) \\
& \times \xi\left(s-\frac{z}{2}, \bar{\chi}\right) \xi\left(s+\frac{z}{2}, \chi\right) y^{s} d s .
\end{aligned}
$$

It is with this equation that we will be working throughout this paper.

\section{Character analogues of the extended version of the Ramanujan-Guinand formula}

Lemma 3.1. For $\operatorname{Re} s>1$ and $\operatorname{Re}(s-\eta)>1$,

$$
L(s, \bar{\chi}) L(s-\eta, \chi)=\sum_{n=1}^{\infty} \frac{\bar{\chi}(n)}{n^{s}} \sum_{d \mid n} \chi^{2}(d) d^{\eta} .
$$

Proof. Since the Dirichlet series for both the $L$-functions converge absolutely under the given hypotheses, using [Apostol 1976, Theorem 11.5], we see that

$$
\begin{aligned}
L(s, \bar{\chi}) L(s-\eta, \chi) & =\sum_{\substack{n=1 \\
(n, q)=1}}^{\infty} \frac{\bar{\chi}(n)}{n^{s}} \sum_{\substack{k=1 \\
(k, q)=1}}^{\infty} \frac{\chi(k)}{k^{s-\eta}}=\sum_{\substack{j=1 \\
(j, q)=1}}^{\infty} \frac{1}{j^{s}} \sum_{\substack{n k=j \\
(k, q)=1}} \bar{\chi}(n) \chi(k) k^{\eta} \\
& =\sum_{\substack{j=1 \\
(j, q)=1}}^{\infty} \frac{\bar{\chi}(j)}{j^{s}} \sum_{\substack{n k=j \\
(k, q)=1}} \frac{\chi^{2}(k)}{\chi(k) \bar{\chi}(k)} k^{\eta}=\sum_{j=1}^{\infty} \frac{\bar{\chi}(j)}{j^{s}} \sum_{n k=j} \chi^{2}(k) k^{\eta},
\end{aligned}
$$

where in the last step, we make use of the fact that $\chi(k) \bar{\chi}(k)=1$ for $(k, q)=1$. 
Proof of Theorem 1.5. Assume that $\chi$ is even. Let $\phi(z, s) \equiv 1$. From (2-1) we see that $f(z, t) \equiv 1$. Using (1-9), (1-8), (1-12), and (1-13), we find that the integral

$$
M(z, \mu, \chi):=\int_{0}^{\infty} \Xi\left(\frac{t+i z}{2}, \bar{\chi}\right) \Xi\left(\frac{t-i z}{2}, \chi\right) \cos \mu t d t
$$

does converge. Using (2-5), we observe that

$$
\begin{aligned}
& M(z, \mu, \chi) \\
& =\frac{1}{i \sqrt{y}} \int_{\frac{1}{2}-i \infty}^{\frac{1}{2}+i \infty} \xi\left(s-\frac{z}{2}, \bar{\chi}\right) \xi\left(s+\frac{z}{2}, \chi\right) y^{s} d s \\
& =\frac{1}{i \sqrt{y}} \int_{\frac{1}{2}-i \infty}^{\frac{1}{2}+i \infty} \Gamma\left(\frac{s}{2}-\frac{z}{4}\right) \Gamma\left(\frac{s}{2}+\frac{z}{4}\right) L\left(s-\frac{z}{2}, \bar{\chi}\right) L\left(s+\frac{z}{2}, \chi\right)\left(\frac{\pi}{q y}\right)^{-s} d s .
\end{aligned}
$$

Since $\operatorname{Re} s=\frac{1}{2}$ and $-1<\operatorname{Re} z<1$, we have $0<\operatorname{Re}(s-z / 2)<1$ and $0<$ $\operatorname{Re}(s+z / 2)<1$. Now replace $s$ by $s-z / 2$ and let $\eta=-z$ in Lemma 3.1. Then, for $\operatorname{Re}(s-z / 2)>1$ and $\operatorname{Re}(s+z / 2)>1$,

$$
L\left(s-\frac{z}{2}, \bar{\chi}\right) L\left(s+\frac{z}{2}, \chi\right)=\sum_{n=1}^{\infty} \frac{\bar{\chi}(n)}{n^{s-z / 2}} \sum_{d \mid n} \chi^{2}(d) d^{-z} .
$$

We wish to shift the line of integration from $\operatorname{Re} s=\frac{1}{2}$ to $\operatorname{Re} s=\frac{3}{2}$ in order to be able to use (3-3) in (3-2). Consider a positively oriented rectangular contour formed by $\left[\frac{1}{2}+i T, \frac{1}{2}-i T\right],\left[\frac{1}{2}-i T, \frac{3}{2}-i T\right],\left[\frac{3}{2}-i T, \frac{3}{2}+i T\right]$ and $\left[\frac{3}{2}+i T, \frac{1}{2}+i T\right]$, where $T$ is any positive real number. The integrand on the extreme right side of (3-2) does not have any pole inside the contour. Also as $T \rightarrow \infty$, the integrals along the horizontal segments $\left[\frac{1}{2}-i T, \frac{3}{2}-i T\right]$ and $\left[\frac{3}{2}+i T, \frac{1}{2}+i T\right]$ tend to zero, which can be seen by using (1-12). Hence, employing Cauchy's residue theorem, letting $T \rightarrow \infty$, using (3-3) in (3-2), and interchanging the order of summation and integration, which is valid because of absolute convergence, we observe that

(3-4) $\quad M(z, \mu, \chi)$

$$
=\frac{1}{i \sqrt{y}} \sum_{n=1}^{\infty} \bar{\chi}(n) n^{z / 2}\left(\sum_{d \mid n} \chi^{2}(d) d^{-z}\right) \int_{\frac{3}{2}-i \infty}^{\frac{3}{2}+i \infty} \Gamma\left(\frac{s}{2}-\frac{z}{4}\right) \Gamma\left(\frac{s}{2}+\frac{z}{4}\right)\left(\frac{n \pi}{q y}\right)^{-s} d s .
$$

But from [Oberhettinger 1974, Formula 11.1, p. 115], for $c=\operatorname{Re} s> \pm \operatorname{Re} v$,

$$
\frac{1}{2 \pi i} \int_{c-i \infty}^{c+i \infty} 2^{s-2} w^{-s} \Gamma\left(\frac{s}{2}-\frac{v}{2}\right) \Gamma\left(\frac{s}{2}+\frac{v}{2}\right) x^{-s} d s=K_{v}(w x) .
$$


Hence, using (3-5) with $c=3 / 2, v=z / 2, w=2$, and $x=n \pi / q y$ in (3-4), we find that

$$
M(z, \mu, \chi)=\frac{8 \pi}{\sqrt{y}} \sum_{n=1}^{\infty} \bar{\chi}(n) n^{z / 2}\left(\sum_{d \mid n} \chi^{2}(d) d^{-z}\right) K_{z / 2}\left(\frac{2 \pi n}{q y}\right) .
$$

Now let $\mu=\frac{1}{2} \log \alpha$ in (3-6) so that $y=e^{2 \mu}$ implies that $y=\alpha$. Then using the fact that $\alpha \beta=1$, we deduce that

$$
\begin{aligned}
\frac{1}{8 \pi} \int_{0}^{\infty} \Xi\left(\frac{t+i z}{2}, \bar{\chi}\right) \Xi & \left.\frac{t-i z}{2}, \chi\right) \cos \left(\frac{1}{2} t \log \alpha\right) d t \\
& =\sqrt{\beta} \sum_{n=1}^{\infty} \bar{\chi}(n) n^{z / 2}\left(\sum_{d \mid n} \chi^{2}(d) d^{-z}\right) K_{\frac{z}{2}}\left(\frac{2 \pi n \beta}{q}\right) .
\end{aligned}
$$

Next, observing that replacing $\alpha$ by $\beta$ and/or simultaneously replacing $\chi$ by $\bar{\chi}$ and $z$ by $-z$ in (3-7) leaves the integral on the left side invariant, we obtain (1-15).

Now consider the case when $\chi$ is odd. Again the convergence of the integral $M(z, \mu, \chi)$ can be seen from (1-12) and (1-13). Following similar steps as in the case of even $\chi$ and using the definition of $\xi(s, \chi)$ from (1-8) for $\chi$ odd, we get

(3-8) $\quad M(z, \mu, \chi)$

$=\frac{q}{i \pi \sqrt{y}} \sum_{n=1}^{\infty} \bar{\chi}(n) n^{z / 2} \sum_{d \mid n} \chi^{2}(d) d^{-z} \int_{\frac{3}{2}-i \infty}^{\frac{3}{2}+i \infty} \Gamma\left(\frac{s}{2}-\frac{z}{4}+\frac{1}{2}\right) \Gamma\left(\frac{s}{2}+\frac{z}{4}+\frac{1}{2}\right)\left(\frac{n \pi}{q y}\right)^{-s} d s$.

Now replacing $s$ by $s+1$ in (3-5), we find that for $c=\operatorname{Re} s> \pm \operatorname{Re} v-1$,

$$
\frac{1}{2 \pi i} \int_{c-i \infty}^{c+i \infty} 2^{s-1} w^{-s-1} \Gamma\left(\frac{s+1}{2}-\frac{v}{2}\right) \Gamma\left(\frac{s+1}{2}+\frac{v}{2}\right) x^{-s} d s=x K_{v}(w x) .
$$

Then using (3-9) with $c=\frac{3}{2}, v=0, w=2$ and $x=n \pi / q y$ in (3-8), we see that

$$
M(z, \mu, \chi)=\frac{8 \pi}{y^{3 / 2}} \sum_{n=1}^{\infty} \bar{\chi}(n) n^{z / 2+1}\left(\sum_{d \mid n} \chi^{2}(d) d^{-z}\right) K_{z / 2}\left(\frac{2 \pi n}{q y}\right) .
$$

Now let $\mu=\frac{1}{2} \log \alpha$ in (3-10) so that $y=e^{2 \mu}$ implies that $y=\alpha$. Then using the fact that $\alpha \beta=1$, we deduce that

$$
\begin{aligned}
\frac{1}{8 \pi} \int_{0}^{\infty} \Xi\left(\frac{t+i z}{2}, \bar{\chi}\right) \Xi\left(\frac{t-i z}{2}, \chi\right) \cos \left(\frac{1}{2} t \log \alpha\right) d t \\
=\beta^{3 / 2} \sum_{n=1}^{\infty} \bar{\chi}(n) n^{z / 2+1}\left(\sum_{d \mid n} \chi^{2}(d) d^{-z}\right) K_{z / 2}\left(\frac{2 \pi n \beta}{q}\right) .
\end{aligned}
$$

Next, observing that replacing $\alpha$ by $\beta$ and/or simultaneously replacing $\chi$ by $\bar{\chi}$ and $z$ by $-z$ in (3-11) leaves the integral on the left side invariant, we obtain (1-15). 
Remark. Letting $z \rightarrow 0$ in Theorem 1.5 gives a new character analogue of the extended version of Koshliakov's formula, that is, Theorem 1.4.

When $\chi$ is real, Theorem 1.5 reduces to the following corollary.

Corollary 3.2. Let $-1<\operatorname{Re} z<1$ and let $\chi$ denote a real, primitive, nonprincipal character modulo $q$. Let the number $b$ be defined as in (1-7). If

$$
F(z, \alpha, \chi)=\alpha^{b+\frac{1}{2}} \sum_{n=1}^{\infty} \chi(n) n^{-\frac{z}{2}+b} \sigma_{z}(n) K_{-z / 2}\left(\frac{2 \pi n \alpha}{q}\right),
$$

then

$$
\begin{aligned}
F(z, \alpha, \chi) & =F(-z, \beta, \chi)=F(-z, \alpha, \chi)=F(z, \beta, \chi) \\
& =\frac{1}{8 \pi} \int_{0}^{\infty} \Xi\left(\frac{t+i z}{2}, \chi\right) \Xi\left(\frac{t-i z}{2}, \chi\right) \cos \left(\frac{1}{2} t \log \alpha\right) d t .
\end{aligned}
$$

The above corollary (without the integral) is equivalent to the special cases, when $\chi$ is real, of the character analogues of the Ramanujan-Guinand formula established in [Berndt et al. 2011, Theorems 3.1 and 4.1].

\section{Inverse Mellin transforms and asymptotic expansions}

We will now evaluate inverse Mellin transforms of some functions and asymptotic expansions of certain other functions used in the later sections.

Lemma 4.1. Let $z \in \mathbb{C}$ be fixed such that $-1<\operatorname{Re} z<1$. For a primitive, nonprincipal character $\chi \bmod q$, let $L(z, a, \chi)$ be defined as in (1-17). Then, for

$$
-\operatorname{Re} \frac{z}{2}<c=\operatorname{Re} s<1+\operatorname{Re} \frac{z}{2}
$$

and $x \in \mathbb{R} \backslash \mathbb{Z}_{<0}$,

$$
\begin{aligned}
\frac{1}{2 \pi i} \int_{c-i \infty}^{c+i \infty} \Gamma\left(s+\frac{z}{2}\right) \Gamma\left(1-s+\frac{z}{2}\right) L\left(1-s+\frac{z}{2}, \chi\right) x^{-s} d s \\
=x^{z / 2} \Gamma(z+1) L(z+1, x, \chi) .
\end{aligned}
$$

Proof. We prove the result only for even characters. The case for odd characters can be proved similarly. We first assume $|x|<1$ and later extend it to any real $x \in \mathbb{R} \backslash \mathbb{Z}_{<0}$ by analytic continuation. Let

$$
-\operatorname{Re}(z / 2)<c=\operatorname{Re} s<1+\operatorname{Re}(z / 2) .
$$

Consider a positively oriented rectangular contour formed by $[c-i T, c+i T]$, $[c+i T,-M+i T],[-M+i T,-M-i T]$, and $[-M-i T, c-i T]$, where $T$ is some positive real number and $M=n-\frac{1}{2}$, where $n$ is a positive integer. Let $s=\sigma+i t$. Among the poles of the function $\Gamma(s+z / 2) \Gamma(1-s+z / 2) L(1-s+z / 2, \chi) x^{-s}$, the only ones that contribute are the poles at $s=-z / 2-m, m \geq 0$. Let $R_{f}(a)$ denote 
the residue of the function $f(s):=\Gamma(s+z / 2) \Gamma(1-s+z / 2) L(1-s+z / 2, \chi) x^{-s}$ at $a$. Then for $m \geq 0$,

$$
\begin{aligned}
& R_{f}\left(-\frac{z}{2}-m\right) \\
& \quad=\lim _{s \rightarrow-z / 2-m}\left(s+\frac{z}{2}+m\right) \Gamma\left(s+\frac{z}{2}\right) \Gamma\left(1-s+\frac{z}{2}\right) L\left(1-s+\frac{z}{2}, \chi\right) x^{-s} \\
& \quad=\frac{(-1)^{m}}{m !} \Gamma(1+z+m) L(1+z+m, \chi) x^{m+z / 2} .
\end{aligned}
$$

From (4-2) and the residue theorem, we have

$$
\begin{aligned}
& {\left[\int_{c-i T}^{c+i T}+\int_{c+i T}^{-M+i T}+\int_{-M+i T}^{-M-i T}+\int_{-M-i T}^{c-i T}\right]} \\
& \Gamma\left(s+\frac{z}{2}\right) \Gamma\left(1-s+\frac{z}{2}\right) L\left(1-s+\frac{z}{2}, \chi\right) x^{-s} d s \\
& \quad=2 \pi i x^{z / 2} \sum_{0 \leq m<M} \frac{(-1)^{m}}{m !} \Gamma(1+z+m) L(1+z+m, \chi) x^{m} .
\end{aligned}
$$

We now estimate the integral along the upper horizontal segment. Using (1-13), we find that for $-M \leq \sigma \leq c$,

$$
L(1-\sigma \pm i T, \chi)=O\left(q^{c+1 / 2} T^{c+1 / 2}\right) .
$$

Hence, for $-M \leq \sigma \leq c$, i.e., $-M-\operatorname{Re} z / 2 \leq \sigma-\operatorname{Re} z / 2 \leq c-\operatorname{Re} z / 2$, we have

$$
\begin{aligned}
L\left(1-\left(\sigma-\operatorname{Re} \frac{z}{2}\right)-i(T-\right. & \left.\left.\operatorname{Im} \frac{z}{2}\right), \chi\right) \\
& =O\left(q^{c-\operatorname{Re} z / 2+1 / 2}\left(T-\operatorname{Im} \frac{z}{2}\right)^{c-\operatorname{Re} z / 2+1 / 2}\right) .
\end{aligned}
$$

By (1-12), we observe that

$$
\left|\Gamma\left(s+\frac{z}{2}\right)\right| \sim \sqrt{2 \pi} e^{-\pi / 2|T+\operatorname{Im} z / 2|} \cdot\left|T+\operatorname{Im} \frac{z}{2}\right|^{\sigma+\operatorname{Re} z / 2-1 / 2}
$$

and

$$
\left|\Gamma\left(1-s+\frac{z}{2}\right)\right| \sim \sqrt{2 \pi} e^{-\frac{\pi}{2}|T-\operatorname{Im} z / 2|} \cdot\left|T-\operatorname{Im} \frac{z}{2}\right|^{-\sigma+\operatorname{Re} z / 2+1 / 2} .
$$

Since $|x|<1$, from (4-5), (4-6), and (4-7), we deduce that

$$
\begin{aligned}
& \left|\int_{c+i T}^{-M+i T} \Gamma\left(s+\frac{z}{2}\right) \Gamma\left(1-s+\frac{z}{2}\right) L\left(1-s+\frac{z}{2}, \chi\right) x^{-s} d s\right| \\
& \leq 2 \pi K_{1}(c+M)|x|^{-c} q^{c-\operatorname{Re} z / 2+1 / 2} e^{-\pi / 2(|T+\operatorname{Im} z / 2|+|T-\operatorname{Im} z / 2|)} \\
& \times\left|T+\operatorname{Im} \frac{z}{2}\right|^{\sigma+\operatorname{Re} z / 2-1 / 2}\left|T-\operatorname{Im} \frac{z}{2}\right|^{c-\sigma+1},
\end{aligned}
$$


where $K_{1}$ is some absolute constant. Hence

$$
\int_{c+i \infty}^{-M+i \infty} \Gamma\left(s+\frac{z}{2}\right) \Gamma\left(1-s+\frac{z}{2}\right) L\left(1-s+\frac{z}{2}, \chi\right) x^{-s} d s=0 .
$$

Similarly for the integral along the lower horizontal segment, using (4-6), (4-7), and the fact that

$L\left(1-\left(\sigma-\operatorname{Re} \frac{z}{2}\right)+i\left(T+\operatorname{Im} \frac{z}{2}\right), \chi\right)=O\left(q^{c-\operatorname{Re} z / 2+1 / 2}\left(T+\operatorname{Im} \frac{z}{2}\right)^{c-\operatorname{Re} z / 2+1 / 2}\right)$,

we observe that

$$
\int_{-M-i \infty}^{c-i \infty} \Gamma(s+z / 2) \Gamma(1-s+z / 2) L(1-s+z / 2, \chi) x^{-s} d s=0 .
$$

Hence, from (4-3), (4-8), and (4-9), it is clear that

$$
\begin{aligned}
& {\left[\int_{c-i \infty}^{c+i \infty}+\int_{-M+i \infty}^{-M-i \infty}\right] \Gamma\left(s+\frac{z}{2}\right) \Gamma\left(1-s+\frac{z}{2}\right) L\left(1-s+\frac{z}{2}, \chi\right) x^{-s} d s} \\
& =2 \pi i x^{z / 2} \sum_{0 \leq m<M} \frac{(-1)^{m}}{m !} \Gamma(1+z+m) L(1+z+m, \chi) x^{m}
\end{aligned}
$$

It remains to evaluate

$$
\int_{-M+i \infty}^{-M-i \infty} \Gamma\left(s+\frac{z}{2}\right) \Gamma\left(1-s+\frac{z}{2}\right) L\left(1-s+\frac{z}{2}, \chi\right) x^{-s} d s .
$$

Using (1-12) and the reflection formula for the gamma function [Temme 1996, Equation (3.5), p. 46], we find that as $|t| \rightarrow \infty$,

$$
\Gamma(-M+i t)=O\left(|t|^{-M-\frac{1}{2}} e^{-\pi|t| / 2}\right) .
$$

Hence, as $|t| \rightarrow \infty$,

$$
\Gamma\left(-M+i t+\frac{z}{2}\right)=O\left(\left|t+\operatorname{Im} \frac{z}{2}\right|^{-M+\operatorname{Re} z / 2-1 / 2} e^{-(\pi / 2)|t+\operatorname{Im} z / 2|}\right) .
$$

Again by (1-12), as $|t| \rightarrow \infty$,

$$
\begin{aligned}
& \left|\Gamma\left(1+M-i t+\frac{z}{2}\right)\right| \\
& \quad=\sqrt{2 \pi} e^{-(\pi / 2)|t-\operatorname{Im} z / 2|} \cdot\left|t-\operatorname{Im} \frac{z}{2}\right|^{M+\operatorname{Re} z / 2+1 / 2}\left(1+O\left(\frac{1}{|t-\operatorname{Im} z / 2|}\right)\right) .
\end{aligned}
$$


Also, $L(1+M-i t+z / 2, \chi)$ is bounded as $\operatorname{Re}(1+M-i t+z / 2)>1$. Hence,

$$
\begin{aligned}
& \left|\int_{-M+i \infty}^{-M-i \infty} \Gamma\left(s+\frac{z}{2}\right) \Gamma\left(1-s+\frac{z}{2}\right) L\left(1-s+\frac{z}{2}, \chi\right) x^{-s} d s\right| \\
& =\left|i \int_{-\infty}^{\infty} \Gamma\left(-M+i t+\frac{z}{2}\right) \Gamma\left(1+M-i t+\frac{z}{2}\right) L\left(1+M-i t+\frac{z}{2}, \chi\right) x^{M-i t} d t\right| \\
& =|x|^{M} \int_{-1}^{1} O(1) d t+|x|^{M} \int_{1}^{ \pm \infty} O\left(\left|t+\operatorname{Im} \frac{z}{2}\right|^{-M+\operatorname{Re} z / 2-1 / 2}\left|t-\operatorname{Im} \frac{z}{2}\right|^{M+\operatorname{Re} z / 2+1 / 2}\right. \\
& \left.\quad \times e^{-(\pi / 2)(|t+\operatorname{Im} z / 2|+|t-\operatorname{Im} z / 2|)}\right) d t \\
& =O\left(|x|^{M}\right) .
\end{aligned}
$$

Since $|x|<1$,

$$
\lim _{M \rightarrow \infty} \int_{-M+i \infty}^{-M-i \infty} \Gamma\left(s+\frac{z}{2}\right) \Gamma\left(1-s+\frac{z}{2}\right) L\left(1-s+\frac{z}{2}, \chi\right) x^{-s} d s=0 .
$$

From (4-10) and (4-13), we finally deduce that

$$
\begin{aligned}
\frac{1}{2 \pi i} \int_{c-i \infty}^{c+i \infty} \Gamma\left(s+\frac{z}{2}\right) \Gamma\left(1-s+\frac{z}{2}\right) L\left(1-s+\frac{z}{2}, \chi\right) x^{-s} d s \\
=x^{z / 2} \sum_{m=0}^{\infty} \frac{(-1)^{m}}{m !} \Gamma(1+z+m) L(1+z+m, \chi) x^{m+z / 2} \\
=x^{z / 2} \Gamma(z+1) \sum_{m=0}^{\infty} \frac{(-1)^{m}}{m !} \frac{\Gamma(1+z+m)}{\Gamma(1+z)} \sum_{k=1}^{\infty} \frac{\chi(k)}{k^{z+m+1}} x^{m} \\
=x^{z / 2} \Gamma(z+1) \sum_{k=1}^{\infty} \frac{\chi(k)}{k^{z+1}} \sum_{m=0}^{\infty} \frac{\Gamma(1+z+m)}{m ! \Gamma(1+z)}\left(\frac{-x}{k}\right)^{m} \\
=x^{z / 2} \Gamma(z+1) \sum_{k=1}^{\infty} \frac{\chi(k)}{k^{z+1}}\left(1+\frac{x}{k}\right)^{-z-1} \\
=x^{z / 2} \Gamma(z+1) \sum_{k=1}^{\infty} \frac{\chi(k)}{(k+x)^{z+1}}=x^{z / 2} \Gamma(z+1) L(z+1, x, \chi),
\end{aligned}
$$

where, in the fourth step, we have utilized the binomial theorem, since $|x|<1$. Since both sides of (4-1) are analytic for any $x \in \mathbb{R} \backslash \mathbb{Z}_{<0}$, the result follows by analytic continuation.

When $z=0$, we get the following corollary. 
Corollary 4.2. For a primitive, nonprincipal character $\chi \bmod q$, let $\psi(a, \chi)$ be defined as in (1-16). Then, for $0<c=\operatorname{Re} s<1$ and $x \in \mathbb{R} \backslash \mathbb{Z}_{<0}$,

$$
\frac{1}{2 \pi i} \int_{c-i \infty}^{c+i \infty} \frac{L(1-s, \chi)}{\sin \pi s} x^{-s} d s=-\frac{1}{\pi} \psi(x, \chi) .
$$

For $j \geq 1$, the generalized Bernoulli numbers $B_{j}(\chi)$ are given by

$$
B_{2 j}(\chi)=\frac{2(-1)^{j-1} G(\bar{\chi})(2 j) !}{q(2 \pi / q)^{2 j}} L(2 j, \chi)
$$

for $\chi$ even and by

$$
B_{2 j-1}(\chi)=\frac{2(-1)^{j-1} i G(\bar{\chi})(2 j-1) !}{q(2 \pi / q)^{2 j-1}} L(2 j-1, \chi)
$$

for $\chi$ odd; see [Berndt 1975, p. 426]. It is also known [Berndt 1975, Corollary 3.4, p. 423] that $B_{2 j-1}(\chi)=0$ when $\chi$ is even and $B_{2 j}(\chi)=0$ when $\chi$ is odd. The asymptotic expansion of $L(z, a, \chi)$ as $|a| \rightarrow \infty$ is given below.

Lemma 4.3. For $\operatorname{Re} z>0$ and $-\pi<\arg a<\pi$, as $|a| \rightarrow \infty$,

$$
L(z, a, \chi) \sim \chi(-1) \sum_{j=1}^{\infty} \frac{B_{j}(\bar{\chi}) \prod_{m=0}^{j-2}(z+m)}{j ! a^{z+j-1}} .
$$

Proof. One takes (4.3) and (4.4) in [Berndt 1975, p. 424], valid for $\chi$ even and odd respectively, substitutes $A=0, B=N, r=1$, and $f(u)=(u+a)^{-z}$, lets $N \rightarrow \infty$, and performs repeated integration by parts on the prevalent integral.

This gives, as a special case, the following asymptotic expansion of $\psi(a, \chi)$ as $|a| \rightarrow \infty$.

Corollary 4.4. For $-\pi<\arg a<\pi$, as $|a| \rightarrow \infty$,

$$
\psi(a, \chi) \sim-\frac{L(0, \chi)}{a}-\chi(-1) \sum_{j=2}^{\infty} \frac{B_{j}(\bar{\chi})}{j a^{j}} .
$$

Proof. Specialize $z=1$ in Lemma 4.3. Observe that $L(1, a, \chi)=-\psi(a, \chi)$. For $\chi$ even, we have $B_{1}(\bar{\chi})=0$. But from [Apostol 1976, p. 268], $L(0, \chi)=0$. This yields (4-16) for $\chi$ even. For $\chi$ odd, we observe from (4-15) that

$$
B_{1}(\bar{\chi})=\frac{i}{\pi} G(\chi) L(1, \bar{\chi}),
$$

and from (1-10), it is easy to see that

$$
L(1, \bar{\chi})=\frac{i \pi}{G(\chi)} L(0, \chi) .
$$

Now (4-16) follows from (4-17) and (4-18). 


\section{Character analogues of Theorem 1.2}

In this section, we prove analogues of Theorem 1.2 for even and odd primitive characters. Then we give character analogues of Ramanujan's transformation formula (Theorem 1.1) as special cases.

Proof of Theorem 1.6. Using Lemma 4.3, one sees that the series involving the functions $L(z, a, \chi)$ in the theorem are convergent. Let

$$
\phi(z, s)=(z+1+2 s) \Gamma\left(\frac{-z-1}{4}+\frac{s}{2}\right) .
$$

From (2-1) and (1-19), we find that $f(z, t / 2)=\frac{1}{2} \Omega(z, t)$. From (2-5), we have

$$
\begin{aligned}
\int_{0}^{\infty} \Omega(z, t) \Xi\left(\frac{t+i z}{2}, \bar{\chi}\right) \Xi\left(\frac{t-i z}{2}, \chi\right) & \cos \mu t d t \\
& =\frac{1}{i \sqrt{y}}(J(z, y, \chi)+J(-z, y, \bar{\chi})),
\end{aligned}
$$

where

$$
J(z, y, \chi):=\int_{\frac{1}{2}-i \infty}^{\frac{1}{2}+i \infty} U(z, s, y, \chi) d s
$$

with

$U(z, s, y, \chi)$

$:=(-z+2 s)(-z+2-2 s) \Gamma\left(\frac{z}{4}+\frac{s}{2}-\frac{1}{2}\right) \Gamma\left(\frac{z}{4}-\frac{s}{2}\right) \xi\left(s-\frac{z}{2}, \bar{\chi}\right) \xi\left(s+\frac{z}{2}, \chi\right) y^{s}$.

Using (1-12) and (1-13), one sees that indeed the integral on the left side of (5-1) converges. We first simplify the integrand in (5-2). Using (1-8) with $b=0$, and then the duplication formula [Temme 1996, Equation (3.4), p. 46] and the reflection formula for the Gamma function in the second equality below, we have

(5-3) $\quad U(z, s, y, \chi)$

$$
\begin{aligned}
&=16\left(\frac{\pi}{q y}\right)^{-s}\left\{\Gamma\left(\frac{z}{4}+\frac{s+1}{2}\right) \Gamma\left(\frac{z}{4}+\frac{s}{2}\right)\right\}\{\left.\Gamma\left(\frac{z}{4}-\frac{s}{2}+1\right) \Gamma\left(\frac{s}{2}-\frac{z}{4}\right)\right\} \\
& \times L\left(s-\frac{z}{2}, \bar{\chi}\right) L\left(s+\frac{z}{2}, \chi\right) \\
&=16\left(\frac{\pi}{q y}\right)^{-s} \cdot \frac{\sqrt{\pi}}{2^{s+z / 2-1}} \Gamma\left(s+\frac{z}{2}\right) \cdot \frac{\pi}{\sin (\pi(s / 2-z / 4))} \\
& \times L\left(s-\frac{z}{2}, \bar{\chi}\right) L\left(s+\frac{z}{2}, \chi\right) .
\end{aligned}
$$

Substituting (1-10) in the form

$$
L\left(s-\frac{z}{2}, \bar{\chi}\right)=\frac{(2 \pi)^{s-z / 2} L(1-s+z / 2, \chi)}{2 q^{s-z / 2-1} G(\chi) \Gamma(s-z / 2) \cos ((\pi / 2)(s-z / 2))}
$$


in (5-3) and then simplifying, we find that

$$
\begin{aligned}
& U(z, s, y, \chi) \\
& =\frac{32 y^{s} 2^{-z} \pi^{(1-z) / 2}}{q^{-z / 2-1} G(\chi)} \Gamma\left(1-s+\frac{z}{2}\right) \Gamma\left(s+\frac{z}{2}\right) L\left(1-s+\frac{z}{2}, \chi\right) L\left(s+\frac{z}{2}, \chi\right) .
\end{aligned}
$$

We wish to shift the line of integration from $\operatorname{Re} s=\frac{1}{2}$ to $\operatorname{Re} s=\frac{3}{2}$ in order to evaluate (5-2), since then $-1<\operatorname{Re} z<1$ implies that

$$
\operatorname{Re}(s+z / 2)>1,
$$

which allows us to use the series representation of $L(s+z / 2, \chi)$. Consider a positively oriented rectangular contour formed by $\left[\frac{1}{2}+i T, \frac{1}{2}-i T\right],\left[\frac{1}{2}-i T, \frac{3}{2}-i T\right]$, $\left[\frac{3}{2}-i T, \frac{3}{2}+i T\right]$, and $\left[\frac{3}{2}+i T, \frac{1}{2}+i T\right]$, where $T$ is any positive real number. The integrand in (5-2) does not have any pole inside the contour since the pole of $\Gamma(1-s+z / 2)$ at $s=1+z / 2$ is canceled by the zero of $L(1-s+z / 2, \chi)$ there. Also as $T \rightarrow \infty$, the integrals along the horizontal segments $\left[\frac{1}{2}-i T, \frac{3}{2}-i T\right]$ and $\left[\frac{3}{2}+i T, \frac{1}{2}+i T\right]$ tend to zero, which can be seen using (1-12). Employing the residue theorem, letting $T \rightarrow \infty$ and using (5-4), we find that

$$
\begin{aligned}
& J(z, y, \chi) \\
& \begin{aligned}
=\frac{32 \cdot 2^{-z} \pi^{(1-z) / 2}}{q^{-z / 2-1} G(\chi)} \int_{\frac{3}{2}-i \infty}^{\frac{3}{2}+i \infty} \Gamma\left(s+\frac{z}{2}\right) \Gamma\left(1-s+\frac{z}{2}\right) \\
\quad \times L\left(1-s+\frac{z}{2}, \chi\right) L\left(s+\frac{z}{2}, \chi\right) y^{s} d s \\
=\frac{32 \cdot 2^{-z} \pi^{(1-z) / 2}}{q^{-z / 2-1} G(\chi)} \sum_{n=1}^{\infty} \frac{\chi(n)}{n^{z / 2}} \int_{\frac{3}{2}-i \infty}^{\frac{3}{2}+i \infty} \Gamma\left(s+\frac{z}{2}\right) \Gamma\left(1-s+\frac{z}{2}\right) \\
\times L\left(1-s+\frac{z}{2}, \chi\right)\left(\frac{n}{y}\right)^{-s} d s .
\end{aligned}
\end{aligned}
$$

Now, in order to use Lemma 4.1 to evaluate the integral in (5-5), we again have to shift the line of integration from $\operatorname{Re} s>\frac{3}{2}$ to $\operatorname{Re} s=d$, where

$$
-\operatorname{Re} z / 2<d<1+\operatorname{Re} z / 2 .
$$

Again, we do not encounter any pole in this process. Hence

$$
J(z, y, \chi)=\frac{64 i 2^{-z} y^{-z / 2} \pi^{(3-z) / 2} \Gamma(z+1)}{q^{-z / 2-1} G(\chi)} \sum_{n=1}^{\infty} \sum_{k=1}^{\infty} \frac{\chi(n) \chi(k)}{(k+n / y)^{z+1}} .
$$

Since $-1<\operatorname{Re}(z)<1$, the other integral, namely $J(-z, y, \bar{\chi})$, can be evaluated by simply replacing $z$ by $-z$ and $\chi$ by $\bar{\chi}$ in (5-6). Now (5-1), (5-6), (1-18), and 
the discussion in the previous line give

$$
\begin{aligned}
& \int_{0}^{\infty} \Omega(z, t) \Xi\left(\frac{t+i z}{2}, \bar{\chi}\right) \Xi\left(\frac{t-i z}{2}, \chi\right) \cos \mu t d t \\
&=\frac{64 \pi^{3 / 2} q}{\sqrt{y}}\left(T\left(z, y^{-1}, \chi\right) \sum_{n=1}^{\infty} \sum_{k=1}^{\infty} \frac{\chi(n) \chi(k)}{(k+n / y)^{z+1}}\right. \\
&\left.\quad+T\left(-z, y^{-1}, \bar{\chi}\right) \sum_{n=1}^{\infty} \sum_{k=1}^{\infty} \frac{\bar{\chi}(n) \bar{\chi}(k)}{(k+n / y)^{-z+1}}\right),
\end{aligned}
$$

where it is easy to see from the fact that $-1<\operatorname{Re} z<1$, from the discussion just preceding the statement of Theorem 1.6, and from Lemma 4.3, that both the double series on the right side of (5-7) converge.

Now let $\mu=\frac{1}{2} \log \alpha$ in (5-7) so that $y=e^{2 \mu}$ implies that $y=\alpha$. Then using the fact that $\alpha \beta=1$ and using (1-17) in the second equality below, we deduce that

$$
\begin{aligned}
& \int_{0}^{\infty} \Omega(z, t) \Xi\left(\frac{t+i z}{2}, \bar{\chi}\right) \Xi\left(\frac{t-i z}{2}, \chi\right) \cos \left(\frac{1}{2} t \log \alpha\right) d t \\
&=64 \pi^{3 / 2} q \sqrt{\beta}\left(T(z, \beta, \chi) \sum_{n=1}^{\infty} \sum_{k=1}^{\infty} \frac{\chi(n) \chi(k)}{(k+n \beta)^{z+1}}\right. \\
&\left.+T(-z, \beta, \bar{\chi}) \sum_{n=1}^{\infty} \sum_{k=1}^{\infty} \frac{\bar{\chi}(n) \bar{\chi}(k)}{(k+n \beta)^{-z+1}}\right) \\
&=64 \pi^{3 / 2} q \sqrt{\beta}\left(T(z, \beta, \chi) \sum_{n=1}^{\infty} \chi(n) L(z+1, n \beta, \chi)\right. \\
&\left.\quad+T(-z, \beta, \bar{\chi}) \sum_{n=1}^{\infty} \bar{\chi}(n) L(-z+1, n \beta, \bar{\chi})\right) .
\end{aligned}
$$

The integral on the extreme left side above is invariant under the transformation $\alpha \rightarrow \beta$ or under the simultaneous application of the transformations

$$
\alpha \rightarrow \beta, \quad \chi \rightarrow \bar{\chi}, \quad z \rightarrow-z
$$

Thus we obtain (1-20).

Next we give an analogue of Ramanujan's transformation formula (Theorem 1.1) for even characters.

Corollary 5.1. For an even, primitive, and nonprincipal character $\chi$ modulo $q$, define $P(\alpha, \chi)$ by

$$
P(\alpha, \chi):=\sqrt{\alpha} \operatorname{Re}\left(G(\chi) \sum_{n=1}^{\infty} \sum_{k=1}^{\infty} \frac{\bar{\chi}(n) \bar{\chi}(k)}{k+n \alpha}\right)=-\sqrt{\alpha} \operatorname{Re}\left(G(\chi) \sum_{n=1}^{\infty} \bar{\chi}(n) \psi(n \alpha, \bar{\chi})\right),
$$


where $\psi(a, \chi)$ is defined in (1-16). Then we have

$$
\begin{aligned}
& P(\alpha, \chi)= P(\beta, \bar{\chi})=P(\alpha, \bar{\chi})=P(\beta, \chi) \\
&=\frac{1}{64 \pi^{3 / 2}} \int_{0}^{\infty}\left(1+t^{2}\right) \Gamma\left(\frac{-1+i t}{4}\right) \Gamma\left(\frac{-1-i t}{4}\right) \\
& \quad \times \Xi\left(\frac{t}{2}, \bar{\chi}\right) \Xi\left(\frac{t}{2}, \chi\right) \cos \left(\frac{1}{2} t \log \alpha\right) d t .
\end{aligned}
$$

Proof. Using Corollary 4.4, we readily see that the double series in the definition of $P(\alpha, \chi)$ converges. Let $z \rightarrow 0$ in (1-20). Then, multiplying both sides by $q$ and using (1-6), we have

$$
\begin{aligned}
\sqrt{\alpha}\left(G(\bar{\chi}) \sum_{n=1}^{\infty}\right. & \left.\sum_{k=1}^{\infty} \frac{\chi(n) \chi(k)}{k+n \alpha}+G(\chi) \sum_{n=1}^{\infty} \sum_{k=1}^{\infty} \frac{\bar{\chi}(n) \bar{\chi}(k)}{k+n \alpha}\right) \\
= & \sqrt{\beta}\left(G(\chi) \sum_{n=1}^{\infty} \sum_{k=1}^{\infty} \frac{\bar{\chi}(n) \bar{\chi}(k)}{k+n \beta}+G(\bar{\chi}) \sum_{n=1}^{\infty} \sum_{k=1}^{\infty} \frac{\chi(n) \chi(k)}{k+n \beta}\right) \\
= & \frac{1}{32 \pi^{3 / 2}} \int_{0}^{\infty}\left(1+t^{2}\right) \Gamma\left(\frac{-1+i t}{4}\right) \Gamma\left(\frac{-1-i t}{4}\right) \\
& \times \Xi\left(\frac{t}{2}, \bar{\chi}\right) \Xi\left(\frac{t}{2}, \chi\right) \cos \left(\frac{1}{2} t \log \alpha\right) d t
\end{aligned}
$$

Each of the first two expressions in (5-9) can be written in two different ways as real parts of a double series. Thus,

$$
\begin{aligned}
\sqrt{\alpha} \operatorname{Re}\left(G(\bar{\chi}) \sum_{n=1}^{\infty} \sum_{k=1}^{\infty} \frac{\chi(n) \chi(k)}{k+n \alpha}\right) \\
=\sqrt{\alpha} \operatorname{Re}\left(G(\chi) \sum_{n=1}^{\infty} \sum_{k=1}^{\infty} \frac{\bar{\chi}(n) \bar{\chi}(k)}{k+n \alpha}\right) \\
=\sqrt{\beta} \operatorname{Re}\left(G(\chi) \sum_{n=1}^{\infty} \sum_{k=1}^{\infty} \frac{\bar{\chi}(n) \bar{\chi}(k)}{k+n \beta}\right) \\
=\sqrt{\beta} \operatorname{Re}\left(G(\bar{\chi}) \sum_{n=1}^{\infty} \sum_{k=1}^{\infty} \frac{\chi(n) \chi(k)}{k+n \beta}\right) \\
=\frac{1}{64 \pi^{3 / 2}} \int_{0}^{\infty}\left(1+t^{2}\right) \Gamma\left(\frac{-1+i t}{4}\right) \Gamma\left(\frac{-1-i t}{4}\right) \\
\quad \times \Xi\left(\frac{t}{2}, \bar{\chi}\right) \Xi\left(\frac{t}{2}, \chi\right) \cos \left(\frac{1}{2} t \log \alpha\right) d t .
\end{aligned}
$$

This implies (5-8).

Moreover, if we start with the integral in Corollary 5.1, evaluate it using (2-5) with $z=0$, and make use of Corollary 4.2 when $\chi$ is even, we obtain the same result 
as in Corollary 5.1, except that the function $P(\alpha, \chi)$ is replaced by the function $F(\alpha, \chi)$ defined by

(5-10) $F(\alpha, \chi):=\sqrt{\alpha} G(\chi) \sum_{n=1}^{\infty} \sum_{k=1}^{\infty} \frac{\bar{\chi}(n) \bar{\chi}(k)}{k+n \alpha}=-\sqrt{\alpha} G(\chi) \sum_{n=1}^{\infty} \bar{\chi}(n) \psi(n \alpha, \bar{\chi})$.

It is then trivial to see that $F(\alpha, \chi)=P(\alpha, \chi)$.

Theorem 1.7 can be analogously proved using Lemma 4.1 for $\chi$ odd. We just note that there we have to take care of the pole of

$$
\Gamma\left(1-s+\frac{1}{2} z\right)
$$

in the integrands of two separate integrals. However, in the calculations that follow later, the two residues turn out to be additive inverses of each other and hence do not contribute anything.

The following is an analogue of Theorem 1.1 (Ramanujan's transformation formula) for odd characters.

Corollary 5.2. For an odd, primitive and, nonprincipal character $\chi$ modulo $q$, define $Q(\alpha, \chi)$ by

$$
Q(\alpha, \chi):=\sqrt{\alpha} \operatorname{Im}\left(G(\chi) \sum_{n=1}^{\infty} \sum_{k=1}^{\infty} \frac{\bar{\chi}(n) \bar{\chi}(k)}{k+n \alpha}\right)=-\sqrt{\alpha} \operatorname{Im}\left(G(\chi) \sum_{n=1}^{\infty} \bar{\chi}(n) \psi(n \alpha, \bar{\chi})\right),
$$

where $\psi(a, \chi)$ is defined as in (1-16). Then we have

$$
\begin{aligned}
& Q(\alpha, \chi)=Q(\beta, \bar{\chi})=Q(\alpha, \bar{\chi})=Q(\beta, \chi) \\
& =\frac{1}{4 \pi^{1 / 2} q} \int_{0}^{\infty} \Gamma\left(\frac{1+i t}{4}\right) \Gamma\left(\frac{1-i t}{4}\right) \\
& \quad \times \Xi\left(\frac{t}{2}, \bar{\chi}\right) \Xi\left(\frac{t}{2}, \chi\right) \cos \left(\frac{1}{2} t \log \alpha\right) d t .
\end{aligned}
$$

Proof. Using Corollary 4.4, we find that the double series in the definition of $Q(\alpha, \chi)$ converges. Let $z \rightarrow 0$ in Theorem 1.7. Multiplying both sides by $-q$ and using (1-5) and (1-6), we observe that

$$
\begin{aligned}
& \sqrt{\alpha}\left(G(\bar{\chi}) \sum_{n=1}^{\infty} \sum_{k=1}^{\infty} \frac{\chi(n) \chi(k)}{k+n \alpha}+G(\chi) \sum_{n=1}^{\infty} \sum_{k=1}^{\infty} \frac{\bar{\chi}(n) \bar{\chi}(k)}{k+n \alpha}\right) \\
& =\sqrt{\beta}\left(G(\chi) \sum_{n=1}^{\infty} \sum_{k=1}^{\infty} \frac{\bar{\chi}(n) \bar{\chi}(k)}{k+n \beta}+G(\bar{\chi}) \sum_{n=1}^{\infty} \sum_{k=1}^{\infty} \frac{\chi(n) \chi(k)}{k+n \beta}\right) \\
& =\frac{i}{2 \pi^{1 / 2} q} \int_{0}^{\infty} \Gamma\left(\frac{1+i t}{4}\right) \Gamma\left(\frac{1-i t}{4}\right) \Xi\left(\frac{t}{2}, \bar{\chi}\right) \Xi\left(\frac{t}{2}, \chi\right) \cos \left(\frac{1}{2} t \log \alpha\right) d t
\end{aligned}
$$


Now, using (1-6) for odd characters to simplify (5-12), we see that

$$
\begin{aligned}
2 i \sqrt{\alpha} \operatorname{Im}(G(\bar{\chi}) & \left.\sum_{n=1}^{\infty} \sum_{k=1}^{\infty} \frac{\chi(n) \chi(k)}{k+n \alpha}\right) \\
& =2 i \sqrt{\alpha} \operatorname{Im}\left(G(\chi) \sum_{n=1}^{\infty} \sum_{k=1}^{\infty} \frac{\bar{\chi}(n) \bar{\chi}(k)}{k+n \alpha}\right) \\
& =2 i \sqrt{\beta} \operatorname{Im}\left(G(\chi) \sum_{n=1}^{\infty} \sum_{k=1}^{\infty} \frac{\bar{\chi}(n) \bar{\chi}(k)}{k+n \beta}\right) \\
& =2 i \sqrt{\beta} \operatorname{Im}\left(G(\bar{\chi}) \sum_{n=1}^{\infty} \sum_{k=1}^{\infty} \frac{\chi(n) \chi(k)}{k+n \beta}\right) \\
& =\frac{i}{2 \pi^{1 / 2} q} \int_{0}^{\infty} \Gamma\left(\frac{1+i t}{4}\right) \Gamma\left(\frac{1-i t}{4}\right) \\
& \times \Xi\left(\frac{t}{2}, \bar{\chi}\right) \Xi\left(\frac{t}{2}, \chi\right) \cos \left(\frac{1}{2} t \log \alpha\right) d t .
\end{aligned}
$$

If we now start with the integral in Corollary 5.2, evaluate it using (2-5) with $z=0$, and make use of Corollary 4.2 when $\chi$ is odd, we obtain the same result as in Corollary 5.2, except that the function $Q(\alpha, \chi)$ is replaced by $-i F(\alpha, \chi)$, where $F(\alpha, \chi)$ is defined in (5-10). It is then trivial to see that $F(\alpha, \chi)=i Q(\alpha, \chi)$.

We separately record the following corollary resulting from the discussion on the previous line and the one succeeding Corollary 5.1.

Corollary 5.3. The sum $F(\alpha, \chi)$ defined in (5-10) is real if $\chi$ is even and purely imaginary if $\chi$ is odd.

\section{Character analogues of the Ramanujan-Hardy-Littlewood conjecture}

In this section, we prove Theorems 1.9 and 1.10. We require [Ahlgren et al. 2002, Lemma 3.1] which states that if $\chi$ is a primitive character of conductor $N$ and $k \geq 2$ is an integer such that $\chi(-1)=(-1)^{k}$,

$$
\frac{(k-2) ! N^{k-2} G(\chi)}{2^{k-1} \pi^{k-2} i^{k-2}} L(k-1, \bar{\chi})=L^{\prime}(2-k, \chi) .
$$

Proof of Theorem 1.9. From [Landau 1905], we have for $\operatorname{Re} s>1$,

$$
\sum_{n=1}^{\infty} \frac{\chi(n) \mu(n)}{n^{s}}=\frac{1}{L(s, \chi)} .
$$

Also, since for $-1<c=\operatorname{Re} s<0$,

$$
\left(1-e^{-x}\right)=-\frac{1}{2 \pi i} \int_{c-i \infty}^{c+i \infty} \Gamma(s) x^{-s} d s
$$


replacing $s$ by $s+1$, we find that for $-2<c<-1$,

$$
\left(1-e^{-x}\right)=-\frac{1}{2 \pi i} \int_{c-i \infty}^{c+i \infty} \Gamma(s+1) x^{-s-1} d s .
$$

Using (6-2) and (6-4), we observe that

$$
\begin{aligned}
& \sum_{n=1}^{\infty} \frac{\chi(n) \mu(n)}{n^{2}} e^{-\pi \alpha^{2} /\left(n^{2} q\right)} \\
& =\frac{1}{L(2, \chi)}-\sum_{n=1}^{\infty} \frac{\chi(n) \mu(n)}{n^{2}}\left(1-e^{-\pi \alpha^{2} /\left(n^{2} q\right)}\right) \\
& =\frac{1}{L(2, \chi)}+\frac{q}{2 \pi^{2} i \alpha^{2}} \int_{c-i \infty}^{c+i \infty} \sum_{n=1}^{\infty} \frac{\chi(n) \mu(n)}{n^{-2 s}} \Gamma(s+1)\left(\frac{\pi \alpha^{2}}{q}\right)^{-s} d s \\
& =\frac{1}{L(2, \chi)}+\frac{q}{2 \pi^{2} i \alpha^{2}} \int_{c-i \infty}^{c+i \infty} \frac{\Gamma(s+1)}{L(-2 s, \chi)}\left(\frac{\pi \alpha^{2}}{q}\right)^{-s} d s,
\end{aligned}
$$

where in the second step above, we interchanged the order of summation and integration, which is valid because of absolute convergence. For $\chi$ odd, (1-10) can be put in the form

$$
\left(\frac{\pi}{q}\right)^{-(2-s) / 2} \Gamma\left(\frac{2-s}{2}\right) L(1-s, \bar{\chi})=\frac{i q^{1 / 2}}{G(\chi)}\left(\frac{\pi}{q}\right)^{-(s+1) / 2} \Gamma\left(\frac{s+1}{2}\right) L(s, \chi) .
$$

Hence

$$
\frac{\Gamma(s+1)}{L(-2 s, \chi)}=\frac{G(\bar{\chi})}{i q^{1 / 2}}\left(\frac{\pi}{q}\right)^{2 s+\frac{1}{2}} \frac{\Gamma\left(\frac{1}{2}-s\right)}{L(2 s+1, \bar{\chi})} .
$$

Substituting (6-6) in (6-5), we observe that

$$
\begin{aligned}
\sum_{n=1}^{\infty} \frac{\chi(n) \mu(n)}{n^{2}} e^{-\pi \alpha^{2} /\left(n^{2} q\right)} & \\
& =\frac{1}{L(2, \chi)}-\frac{G(\bar{\chi})}{2 \pi^{3 / 2} \alpha^{2}} \int_{c-i \infty}^{c+i \infty} \frac{\Gamma\left(\frac{1}{2}-s\right)}{L(2 s+1, \bar{\chi})}\left(\frac{q \alpha^{2}}{\pi}\right)^{-s} d s .
\end{aligned}
$$

We wish to shift the line of integration from $\operatorname{Re} s=c,-2<c<-1$, to $\operatorname{Re} s=\lambda$, where $\frac{1}{2}<\lambda<\frac{3}{2}$. Consider a positively oriented rectangular contour formed by $[c-i T, \lambda-i T],[\lambda-i T, \lambda+i T],[\lambda+i T, c+i T]$, and $[c+i T, c-i T]$, where $T$ is any positive real number. Let $\rho=\delta+i \gamma$ denote a nontrivial zero of $L(s, \bar{\chi})$. Let $T \rightarrow \infty$ through values such that $|T-\gamma|>\exp \left(-A_{1} \gamma / \log \gamma\right)$ for every ordinate $\gamma$ of a zero of $L(s, \bar{\chi})$. It is known [Davenport 2000, p. 102] that for $t$ not coinciding 
with the ordinate $\gamma$ of a zero, and $-1 \leq \sigma \leq 2$,

$$
\frac{L^{\prime}(s, \bar{\chi})}{L(s, \bar{\chi})}=\sum_{|t-\gamma| \leq 1} \frac{1}{s-\rho}+O(\log (q(|t|+2))),
$$

from which we can conclude that

$$
\log L(s, \bar{\chi})=\sum_{|t-\gamma| \leq 1} \log (s-\rho)+O(\log (q(|t|+2))) .
$$

Taking real parts in (6-8) gives

$$
\begin{aligned}
\log |L(s, \bar{\chi})| & =\sum_{|t-\gamma| \leq 1} \log |s-\rho|+O(\log (q(|t|+2))) \\
& \geq \sum_{|t-\gamma| \leq 1} \log |t-\gamma|+O(\log (q(|t|+2))) .
\end{aligned}
$$

Hence, from (6-9), we have

$$
\begin{aligned}
\log |L(\sigma+i T, \bar{\chi})| & \geq-\sum_{|T-\gamma| \leq 1} A_{1} \gamma / \log \gamma+O(\log (q(|T|+2))) \\
& >-A_{2} T,
\end{aligned}
$$

where $A_{2}<\pi / 4$ if $A_{1}$ is small enough and $T>T_{0}$ for some fixed $T_{0}$. From (6-10), we see that

$$
\left|\frac{1}{L(2 s+1, \bar{\chi})}\right|<e^{A_{3} T},
$$

where $A_{3}<\pi / 2$. Using (1-12) and (6-11), we observe that as $T \rightarrow \infty$ through the above values, the integrals along the horizontal segments tend to zero. Now let $(\rho-1) / 2:=\delta+i \gamma$ denote a nontrivial zero of $L(2 s+1, \bar{\chi})$. Let $R_{f}(a)$ denote the residue at $a$ of the function

$$
f(s):=\frac{\Gamma\left(\frac{1}{2}-s\right)}{L(2 s+1, \bar{\chi})}\left(\frac{q \alpha^{2}}{\pi}\right)^{-s} .
$$

The nontrivial zeros of $L(2 s+1, \bar{\chi})$ lie in the critical strip $-\frac{1}{2}<\operatorname{Re} s<0$, whereas the trivial zeros are at $-1,-2,-3, \ldots$ Also, $\Gamma\left(\frac{1}{2}-s\right)$ has poles at $\frac{1}{2}, \frac{3}{2}, \frac{5}{2}, \ldots$ Then the residue theorem yields

$$
\int_{c-i \infty}^{c+i \infty} \frac{\Gamma\left(\frac{1}{2}-s\right)}{L(2 s+1, \bar{\chi})}\left(\frac{q \alpha^{2}}{\pi}\right)^{-s} d s
$$

$=\int_{\lambda-i \infty}^{\lambda+i \infty} \frac{\Gamma\left(\frac{1}{2}-s\right)}{L(2 s+1, \bar{\chi})}\left(\frac{q \alpha^{2}}{\pi}\right)^{-s} d s-2 \pi i\left(R_{f}(-1)+\sum_{\rho} R_{f}\left(\frac{\rho-1}{2}\right)+R_{f}\left(\frac{1}{2}\right)\right)$, 
where

$$
R_{f}(-1)=\lim _{s \rightarrow-1}(s+1) \frac{\Gamma\left(\frac{1}{2}-s\right)}{L(2 s+1, \bar{\chi})}\left(\frac{q \alpha^{2}}{\pi}\right)^{-s}=\frac{\alpha^{2} q}{4 \sqrt{\pi} L^{\prime}(-1, \bar{\chi})},
$$

$$
\begin{aligned}
R_{f}\left(\frac{\rho-1}{2}\right) & =\lim _{s \rightarrow(\rho-1) / 2}\left(s-\frac{\rho-1}{2}\right) \frac{\Gamma\left(\frac{1}{2}-s\right)}{L(2 s+1, \bar{\chi})}\left(\frac{q \alpha^{2}}{\pi}\right)^{-s} \\
& =\frac{\Gamma((2-\rho) / 2)}{2 L^{\prime}(\rho, \bar{\chi})}\left(\frac{\pi}{q \alpha^{2}}\right)^{(\rho-1) / 2}
\end{aligned}
$$

$$
R_{f}(1 / 2)=-\frac{\sqrt{\pi}}{\alpha \sqrt{q} L(2, \bar{\chi})} \text {. }
$$

Of course, here we have assumed that the nontrivial zeros of $L(2 s+1, \bar{\chi})$ are all simple and that $\sum_{\rho} R_{f}((\rho-1) / 2)$ converges, since the aforementioned discussion regarding the integrals along the horizontal segments tending to zero as $T \rightarrow \infty$ through the chosen sequence does not imply the convergence of $\sum_{\rho} R_{f}((\rho-1) / 2)$ in the ordinary sense. It only means that the series converges only when we bracket the terms in such a way that the two terms for which

$$
\left|\gamma-\gamma^{\prime}\right|<\exp \left(\frac{-A_{1}|\gamma|}{\log (|\gamma|+2)}\right)+\exp \left(\frac{-A_{1}\left|\gamma^{\prime}\right|}{\log \left(\left|\gamma^{\prime}\right|+2\right)}\right)
$$

are included in the same bracket. Using (6-2) and interchanging the order of summation and integration, which is valid because of absolute convergence, we obtain

$$
\begin{aligned}
\int_{\lambda-i \infty}^{\lambda+i \infty} \frac{\Gamma\left(\frac{1}{2}-s\right)}{L(2 s+1, \bar{\chi})} & \left(\frac{q \alpha^{2}}{\pi}\right)^{-s} d s \\
& =\sum_{n=1}^{\infty} \frac{\bar{\chi}(n) \mu(n)}{n} \int_{\lambda-i \infty}^{\lambda+i \infty} \Gamma\left(\frac{1}{2}-s\right)\left(\frac{q \alpha^{2} n^{2}}{\pi}\right)^{-s} d s \\
& =\frac{\sqrt{\pi}}{\alpha \sqrt{q}} \sum_{n=1}^{\infty} \frac{\bar{\chi}(n) \mu(n)}{n^{2}} \int_{d-i \infty}^{d+i \infty} \Gamma(s)\left(\frac{\pi}{\alpha^{2} n^{2} q}\right)^{-s} d s
\end{aligned}
$$

where in the penultimate line, we have made the change of variable $s \rightarrow \frac{1}{2}-s$ so that $-1<d<0$. Thus, Equations (6-3) and (6-12)-(6-16) imply

$$
\begin{aligned}
& \int_{c-i \infty}^{c+i \infty} \frac{\Gamma\left(\frac{1}{2}-s\right)}{L(2 s+1, \bar{\chi})}\left(\frac{q \alpha^{2}}{\pi}\right)^{-s} d s=-\frac{2 \pi^{3 / 2} i}{\alpha \sqrt{q}} \sum_{n=1}^{\infty} \frac{\bar{\chi}(n) \mu(n)}{n^{2}}\left(1-e^{-\pi /\left(\alpha^{2} n^{2} q\right)}\right) \\
& -2 \pi i\left(\frac{\alpha^{2} q}{4 \sqrt{\pi} L^{\prime}(-1, \bar{\chi})}+\sum_{\rho} \frac{\Gamma((2-\rho) / 2)}{2 L^{\prime}(\rho, \bar{\chi})}\left(\frac{\pi}{q \alpha^{2}}\right)^{(\rho-1) / 2}-\frac{\sqrt{\pi}}{\alpha \sqrt{q} L(2, \bar{\chi})}\right) .
\end{aligned}
$$


From (6-7), (6-17), and the fact that $\alpha \beta=1$ and $\sqrt{G(\chi) G(\bar{\chi})}=i \sqrt{q}$, we find that

$$
\begin{aligned}
& \alpha \sqrt{\alpha} \sqrt{G(\chi)} \sum_{n=1}^{\infty} \frac{\chi(n) \mu(n)}{n^{2}} e^{-\pi \alpha^{2} /\left(n^{2} q\right)} \\
= & \frac{\alpha \sqrt{\alpha} \sqrt{G(\chi)}}{L(2, \chi)}-\frac{\beta \sqrt{\beta} \sqrt{G(\bar{\chi})}}{L(2, \bar{\chi})}+\beta \sqrt{\beta} \sqrt{G(\bar{\chi})} \sum_{n=1}^{\infty} \frac{\bar{\chi}(n) \mu(n)}{n^{2}} e^{-\pi \beta^{2} /\left(n^{2} q\right)} \\
- & \frac{\alpha \sqrt{\alpha} q^{3 / 2} \sqrt{G(\bar{\chi})}}{4 \pi L^{\prime}(-1, \bar{\chi})}-\frac{q \sqrt{G(\bar{\chi})}}{2 \pi \sqrt{\beta}} \sum_{\rho} \frac{\Gamma((2-\rho) / 2)}{L^{\prime}(\rho, \bar{\chi})}\left(\frac{\pi}{q}\right)^{\rho / 2} \beta^{\rho}+\frac{\beta \sqrt{\beta} \sqrt{G(\bar{\chi})}}{L(2, \bar{\chi})} .
\end{aligned}
$$

Applying (6-1) with $N=q$ and $k=3$, and replacing $\chi$ by $\bar{\chi}$ gives

$$
\frac{1}{L^{\prime}(-1, \bar{\chi})}=\frac{4 \pi i}{q G(\bar{\chi}) L(2, \chi)} \text {. }
$$

Thus (6-18) and (6-19) yield

$$
\begin{aligned}
& \alpha \sqrt{\alpha} \sqrt{G(\chi)} \sum_{n=1}^{\infty} \frac{\chi(n) \mu(n)}{n^{2}} e^{-\pi \alpha^{2} /\left(n^{2} q\right)} \\
& -\beta \sqrt{\beta} \sqrt{G(\bar{\chi})} \sum_{n=1}^{\infty} \frac{\bar{\chi}(n) \mu(n)}{n^{2}} e^{-\pi \beta^{2} /\left(n^{2} q\right)} \\
& =-\frac{q \sqrt{G(\bar{\chi})}}{2 \pi \sqrt{\beta}} \sum_{\rho} \frac{\Gamma((2-\rho) / 2)}{L^{\prime}(\rho, \bar{\chi})}\left(\frac{\pi}{q}\right)^{\rho / 2} \beta^{\rho} .
\end{aligned}
$$

Switching the roles of $\alpha$ and $\beta$ and those of $\chi$ and $\bar{\chi}$ gives

$$
\begin{aligned}
\frac{q \sqrt{G(\chi)}}{2 \pi \sqrt{\alpha}} \sum_{\rho} \frac{\Gamma((2-\rho) / 2)}{L^{\prime}(\rho, \chi)} & \left(\frac{\pi}{q}\right)^{\rho / 2} \alpha^{\rho} \\
& +\frac{q \sqrt{G(\bar{\chi})}}{2 \pi \sqrt{\beta}} \sum_{\rho} \frac{\Gamma((2-\rho) / 2)}{L^{\prime}(\rho, \bar{\chi})}\left(\frac{\pi}{q}\right)^{\rho / 2} \beta^{\rho}=0 .
\end{aligned}
$$

Finally (6-20) and (6-21) give (1-24) upon simplification.

Remark. The approach used above for proving that the integrals along the horizontal segments tend to zero as $T \rightarrow \infty$ through the chosen sequence is adapted from [Titchmarsh 1986, p. 219].

To prove Theorem 1.10, we require the following lemma.

Lemma 6.1.

$$
\sum_{n=1}^{\infty} \frac{\chi(n) \mu(n)}{n}=\frac{1}{L(1, \chi)} .
$$


Proof. Dividing $n$ into its residue classes $\bmod q$ by letting $n=q r+b, 0 \leq r<\infty$, $0 \leq b \leq q-1$, we find that since $\chi$ has period $q$,

$$
\sum_{n=1}^{\infty} \frac{\chi(n) \mu(n)}{n}=\sum_{r=0}^{\infty} \sum_{b=0}^{q-1} \frac{\chi(b) \mu(q r+b)}{q r+b}=\sum_{b=0}^{q-1} \chi(b) \sum_{r=0}^{\infty} \frac{\mu(q r+b)}{q r+b} .
$$

The series $\sum_{r=0}^{\infty} \mu(q r+b) /(q r+b)$ was first studied by Kluyver [1904] and its convergence was proved by Landau [1905]. In fact, Landau gave an explicit representation for this series in terms of a finite sum consisting of $L$-functions. Thus (6-22) implies convergence of $\sum_{n=1}^{\infty} \chi(n) \mu(n) / n$. Then using (6-2) and an analogue of Abel's theorem for power series, we see that

$$
\sum_{n=1}^{\infty} \frac{\chi(n) \mu(n)}{n}=\lim _{s \rightarrow 1} \sum_{n=1}^{\infty} \frac{\chi(n) \mu(n)}{n^{s}}=\lim _{s \rightarrow 1} \frac{1}{L(s, \chi)}=\frac{1}{L(1, \chi)} .
$$

Proof of Theorem 1.10. The proof is very similar to that of Theorem 1.9 and hence we omit the details. However we note that Lemma 6.1, (1-10) in the form [Davenport 2000, p. 69]

$$
\pi^{-(1-s) / 2} q^{(1-s) / 2} \Gamma\left(\frac{1-s}{2}\right) L(1-s, \bar{\chi})=\frac{q^{1 / 2}}{G(\chi)} \pi^{-s / 2} q^{s / 2} \Gamma\left(\frac{s}{2}\right) L(s, \chi),
$$

and (6-1) with $N=q$ and $k=2$ are used in the proof.

\section{Open problems}

Following are some open problems with which we will conclude.

(1) We have indirectly given the proof of the fact that function $F(\alpha, \chi)$ defined in (5-10) is real (respectively purely imaginary) when $\chi$ is even (respectively odd). Prove this directly; that is, without using Corollaries 5.1 and 5.2 and the integrals in those corollaries.

(2) Since (1-23) is of the form $F(\alpha)=F(\beta)$, where $\alpha \beta=1$, it is natural to ask if there exists an integral representation involving the Riemann $\Xi$-function equal to the two expressions in (1-23). Finding an integral representation for either side of (1-23) may shed light on the convergence of

$$
\sum_{\rho} \frac{\Gamma((1-\rho) / 2) a^{\rho}}{\zeta^{\prime}(\rho)}
$$

provided, of course, that the integral converges in the first place. It should be remarked here that Hardy and Littlewood [1916, p. 161] have shown that the relation

$$
P(y)=O\left(y^{-\frac{1}{4}+\delta}\right),
$$


where $P(y)=\sum_{n=1}^{\infty}(-y)^{n} /(n ! \zeta(2 n+1))$ can be derived from (1-23) if we assume the Riemann hypothesis and the absolute convergence of

$$
\sum_{\rho} \frac{\Gamma((1-\rho) / 2)}{\zeta^{\prime}(\rho)}
$$

They have further shown that (7-1) is a necessary and sufficient condition for the Riemann hypothesis to be true.

Similarly, it is natural to ask if the expressions in (1-24) and (1-25) have integral representations involving $\Xi(t / 2, \chi)$.

\section{Acknowledgements}

The author thanks Professor Bruce C. Berndt for his constant support, careful reading of the manuscript and for several helpful comments, and to Professors Alexandru Zaharescu, Kevin Ford, and Adolf Hildebrand for their help. He also thanks Becky S. Burner, Timothy W. Cole, and Margaret A. Lewis from the Mathematics library at University of Illinois at Urbana-Champaign, and Anton Lukyanenko for their great help in locating the Russian references and citations. He thanks Reetesh Ranjan for numerically verifying some of the identities by programming in Fortran. The author also thanks the referee for his valuable suggestions which improved the quality of this paper.

\section{References}

[Ahlgren et al. 2002] S. Ahlgren, B. C. Berndt, A. J. Yee, and A. Zaharescu, "Integrals of Eisenstein series and derivatives of L-functions", Int. Math. Res. Not. 2002:32 (2002), 1723-1738. MR 2003f:11056 Zbl 1086.11023

[Apostol 1972] T. M. Apostol, "Dirichlet $L$-functions and primitive characters", Proc. Amer. Math. Soc. 31:2 (1972), 384-386. MR 44 \#2717 Zbl 0239.10023

[Apostol 1976] T. M. Apostol, Introduction to analytic number theory, Springer, New York, 1976. MR 55 \#7892 Zbl 0335.10001

[Berndt 1970] B. C. Berndt, "Identities involving the coefficients of a class of Dirichlet series, IV", Trans. Amer. Math. Soc. 149:1 (1970), 179-185. MR 41 \#5309 Zbl 0207.05504

[Berndt 1975] B. C. Berndt, "Character analogues of the Poisson and Euler-MacLaurin summation formulas with applications", J. Number Theory 7:4 (1975), 413-445. MR 52 \#3075 Zbl 0316.10023

[Berndt 1998] B. C. Berndt, Ramanujan's notebooks, V, Springer, New York, 1998. MR 99f:11024 Zbl 0886.11001

[Berndt and Dixit 2010] B. C. Berndt and A. Dixit, "A transformation formula involving the gamma and Riemann zeta functions in Ramanujan's lost notebook", pp. 199-210 in The legacy of Alladi Ramakrishnan in the mathematical sciences, edited by K. Alladi et al., Springer, New York, 2010. MR 2011m:11171

[Berndt et al. 2008] B. C. Berndt, Y. Lee, and J. Sohn, "Koshliakov's formula and Guinand's formula in Ramanujan's lost notebook", pp. 21-42 in Surveys in number theory, edited by K. Alladi, Dev. Math. 17, Springer, New York, 2008. MR 2010a:33011 Zbl 1183.33007 
[Berndt et al. 2011] B. C. Berndt, A. Dixit, and J. Sohn, "Character analogues of theorems of Ramanujan, Koshliakov and Guinand", Adv. in Appl. Math. 46:1-4 (2011), 54-70. MR 2012c:33017 Zbl 1216.33012

[Bogolyubov et al. 1990] N. N. Bogolyubov, L. D. Faddeev, A. Y. Ishlinskiŭ, V. N. Koshlyakov, and Y. A. Mitropol'skiı̌, "Nikolai Sergeevich Koshlyakov (on the centenary of his birth)", Uspekhi Mat. Nauk 45:4 (1990), 173-176. In Russian; translated in Russian Math. Surveys 45:4 (1990), 197-202. MR 91i:01064

[Davenport 2000] H. Davenport, Multiplicative number theory, 3rd ed., Grad. Texts in Math. 74, Springer, New York, 2000. MR 2001f:11001 Zbl 1002.11001

[Dixit 2010] A. Dixit, "Series transformations and integrals involving the Riemann $\Xi$-function", $J$. Math. Anal. Appl. 368:1 (2010), 358-373. MR 2011h:11091 Zbl 1206.11104

[Dixit 2011a] A. Dixit, "Analogues of a transformation formula of Ramanujan", Int. J. Number Theory 7:5 (2011), 1151-1172. MR 2825965 Zbl 1229.11113

[Dixit 2011b] A. Dixit, "Transformation formulas associated with integrals involving the Riemann E-function”, Monatsh. Math. 164:2 (2011), 133-156. Zbl 05973790

[Ferrar 1936] W. L. Ferrar, "Some solutions of the equation $F(t)=F\left(t^{-1}\right)$ ", J. London Math. Soc. 11:2 (1936), 99-103. Zbl 0013.39204

[Guinand 1941] A. P. Guinand, "On Poisson's summation formula", Ann. of Math. (2) 42:3 (1941), 591-603. MR 3,109e Zbl 0025.33802

[Guinand 1955] A. P. Guinand, "Some rapidly convergent series for the Riemann $\xi$-function", Quart. J. Math. Oxford Ser. (2) 6:1 (1955), 156-160. MR 17,16a Zbl 0065.27703

[Hardy 1915] G. H. Hardy, "Note by Mr. G. H. Hardy on the preceding paper", Quart. J. Math. 46 (1915), 260-261.

[Hardy and Littlewood 1916] G. H. Hardy and J. E. Littlewood, "Contributions to the theory of the Riemann zeta-function and the theory of the distribution of primes", Acta Math. 41:1 (1916), 119-196. MR 1555148 Zbl 46.0498.01

[Kluyver 1904] J. C. Kluyver, "Series derived from the series $\sum \frac{\mu(m)}{m}$ ", Koningl. Akad. Wetensch. Amsterdam Proc. Sect. Sci. 6 (1904), 305-312.

[Koshlyakov 1928] N. S. Koshlyakov, “On Voronoï’s sum-formula”, Mess. Math. 58 (1928), 30-32. Zbl 54.0197.01

[Koshlyakov 1934a] N. S. Koshlyakov, "On a general summation formula and its applications", $C$. R. (Dokl.) Acad. Sci. URSS 4 (1934), 187-191. In Russian. Zbl 0010.30502

[Koshlyakov 1934b] N. S. Koshlyakov, "Some identities in quadratic fields", C. R. (Dokl.) Acad. Sci. URSS 2:9 (1934), 527-531. In Russian. Zbl 0009.34004

[Koshlyakov 1934c] N. S. Koshlyakov, "Some integral representations of the square of Riemann's function $\Xi(t)$ ”, C. R. (Dokl.) Acad. Sci. URSS 2 (1934), 401-405. In Russian. Zbl 0009.19801

[Koshlyakov 1936] N. S. Koshlyakov, "On an extension of some formulae of Ramanujan”, Proc. London Math. Soc. 41:1 (1936), 26-32. Zbl 0013.35402

[Koshlyakov 1937] N. S. Koshlyakov, "On a transformation of definite integrals and its application to the theory of Riemann's function $\zeta(s)$ ", C.R. (Dokl.) Acad. Sci. URSS 15 (1937), 3-8. Zbl 0017. 01403

[Koshlyakov 1949] N. S. Sergeev, A study of a class of transcendental functions defined by the generalized Riemann equation, Izdat. Akad. Nauk SSSR, Moscow, 1949. In Russian. MR 13,24d Zbl 0045.18401 
[Koshlyakov 1954] N. S. Koshlyakov, "Investigation of some questions of the analytic theory of a rational and quadratic field, I", Izvestiya Akad. Nauk SSSR. Ser. Mat. 18:2 (1954), 113-144. In Russian. MR 16,15d

[Landau 1905] E. Landau, "Remarks on the paper of Mr. Kluyver on page 305 of Vol. VI: Series derived from the series $\sum \frac{\mu(m)}{m}$," Koningl. Akad. Wetensch. Amsterdam Proc. Sect. Sci. 7 (1905), 66-77.

[Ma et al. 2010] R. Ma, Y. Yi, and Y. Zhang, "On the mean value of the generalized Dirichlet $L$ functions”, Czechoslovak Math. J. 60:3 (2010), 597-620. MR 2011j:11162 Zbl 1224.11077

[Oberhettinger 1974] F. Oberhettinger, Tables of Mellin transforms, Springer, New York, 1974. MR 50 \#5376 Zbl 0289.44003

[Paris and Kaminski 2001] R. B. Paris and D. Kaminski, Asymptotics and Mellin-Barnes integrals, Encyclopedia Math. Appl. 85, Cambridge University Press, Cambridge, 2001. MR 2002h:33001 Zbl 0983.41019

[Ramanujan 1915] S. Ramanujan, "New expressions for Riemann's functions $\xi(s)$ and $\Xi(t)$ ", Quart. J. Math. 46 (1915), 253-261. MR 2280857 Zbl 45.1343.05

[Ramanujan 1927] S. Ramanujan, Collected papers of Srinivasa Ramanujan, edited by G. H. Hardy et al., Cambridge University Press, Cambridge, 1927. Reprinted by the Amer. Math. Soc., Providence, RI, 2000. MR 2008b:11002 Zbl 53.0030.02

[Ramanujan 1957] S. Ramanujan, Notebooks, 1, 2, Tata Institute of Fundamental Research, Bombay, 1957. MR 20 \#6340 Zbl 0138.24201

[Ramanujan 1988] S. Ramanujan, The lost notebook and other unpublished papers, Springer, Berlin, 1988. MR 89j:01078 Zbl 0639.01023

[Temme 1996] N. M. Temme, Special functions: an introduction to the classical functions of mathematical physics, Wiley, New York, 1996. MR 97e:33002 Zbl 0856.33001

[Titchmarsh 1986] E. C. Titchmarsh, The theory of the Riemann zeta-function, 2nd ed., edited by D. R. Heath-Brown, Clarendon, Oxford, 1986. MR 88c:11049 Zbl 0601.10026

Received February 13, 2011.

ATUl DiXIT

DEPARTMENT OF MATHEMATICS

UNIVERSITY OF ILLINOIS AT URBANA-CHAMPAIGN

1409 WEST GREEN STREET

URBANA, ILLINOIS 61801

UNITED STATES

aadixit2@illinois.edu

http://www.math.uiuc.edu/ aadixit2/ 


\title{
PACIFIC JOURNAL OF MATHEMATICS
}

\author{
http://pacificmath.org \\ Founded in 1951 by \\ E. F. Beckenbach (1906-1982) and F. Wolf (1904-1989)
}

\section{EDITORS}

V. S. Varadarajan (Managing Editor)

Department of Mathematics

University of California

Los Angeles, CA 90095-1555

pacific@math.ucla.edu

Vyjayanthi Chari

Department of Mathematics

University of California

Riverside, CA 92521-0135

chari@math.ucr.edu

\section{Robert Finn}

Department of Mathematics Stanford University

Stanford, CA 94305-2125

finn@math.stanford.edu

Kefeng Liu

Department of Mathematics

University of California

Los Angeles, CA 90095-1555

liu@math.ucla.edu
Darren Long

Department of Mathematics

University of California

Santa Barbara, CA 93106-3080

long@math.ucsb.edu

Jiang-Hua Lu

Department of Mathematics

The University of Hong Kong

Pokfulam Rd., Hong Kong jhlu@maths.hku.hk

Alexander Merkurjev

Department of Mathematics

University of California

Los Angeles, CA 90095-1555

merkurev@math.ucla.edu
Sorin Popa

Department of Mathematics University of California

Los Angeles, CA 90095-1555 popa@math.ucla.edu

Jie Qing

Department of Mathematics

University of California

Santa Cruz, CA 95064

qing@cats.ucsc.edu

Jonathan Rogawski

Department of Mathematics

University of California

Los Angeles, CA 90095-1555

jonr@math.ucla.edu

\section{PRODUCTION}

pacific@math.berkeley.edu

\section{SUPPORTING INSTITUTIONS}

ACADEMIA SINICA, TAIPEI

CALIFORNIA INST. OF TECHNOLOGY INST. DE MATEMÁTICA PURA E APLICADA KEIO UNIVERSITY

MATH. SCIENCES RESEARCH INSTITUTE NEW MEXICO STATE UNIV.

OREGON STATE UNIV.

\author{
STANFORD UNIVERSITY \\ UNIV. OF BRITISH COLUMBIA \\ UNIV. OF CALIFORNIA, BERKELEY \\ UNIV. OF CALIFORNIA, DAVIS \\ UNIV. OF CALIFORNIA, LOS ANGELES \\ UNIV. OF CALIFORNIA, RIVERSIDE \\ UNIV. OF CALIFORNIA, SAN DIEGO \\ UNIV. OF CALIF., SANTA BARBARA
}

\author{
UNIV. OF CALIF., SANTA CRUZ \\ UNIV. OF MONTANA \\ UNIV. OF OREGON \\ UNIV. OF SOUTHERN CALIFORNIA \\ UNIV. OF UTAH \\ UNIV. OF WASHINGTON \\ WASHINGTON STATE UNIVERSITY
}

These supporting institutions contribute to the cost of publication of this Journal, but they are not owners or publishers and have no responsibility for its contents or policies.

See inside back cover or pacificmath.org for submission instructions.

The subscription price for 2012 is US \$420/year for the electronic version, and \$485/year for print and electronic.

Subscriptions, requests for back issues from the last three years and changes of subscribers address should be sent to Pacific Journal of Mathematics, P.O. Box 4163, Berkeley, CA 94704-0163, U.S.A. Prior back issues are obtainable from Periodicals Service Company, 11 Main Street, Germantown, NY 12526-5635. The Pacific Journal of Mathematics is indexed by Mathematical Reviews, Zentralblatt MATH, PASCAL CNRS Index, Referativnyi Zhurnal, Current Mathematical Publications and the Science Citation Index.

The Pacific Journal of Mathematics (ISSN 0030-8730) at the University of California, c/o Department of Mathematics, 969 Evans Hall, Berkeley, CA 94720-3840, is published monthly except July and August. Periodical rate postage paid at Berkeley, CA 94704, and additional mailing offices. POSTMASTER: send address changes to Pacific Journal of Mathematics, P.O. Box 4163, Berkeley, CA 94704-0163.

PJM peer review and production are managed by EditFLOW ${ }^{\mathrm{TM}}$ from Mathematical Sciences Publishers.

PUBLISHED BY PACIFIC JOURNAL OF MATHEMATICS

at the University of California, Berkeley 94720-3840

A NON-PROFIT CORPORATION

Typeset in LATEX

Copyright $(02012$ by Pacific Journal of Mathematics 


\section{PACIFIC JOURNAL OF MATHEMATICS}

Volume $255 \quad$ No. $2 \quad$ February 2012

On the local Langlands correspondences of DeBacker-Reeder and

Reeder for $\operatorname{GL}(\ell, F)$, where $\ell$ is prime

MOSHE ADRIAN

$R$-groups and parameters

281

DUBRAVKA BAN and DAVID GOLDBERG

Finite-volume complex-hyperbolic surfaces, their toroidal

305

compactifications, and geometric applications

LUCA FABRIZIO Di CERBO

Character analogues of Ramanujan-type integrals involving the Riemann 317 $\Xi$-function

ATUl DiXIT

Spectral theory for linear relations via linear operators

DANA GHEORGHE and FLORIAN-HORIA VASILESCU

Homogeneous links and the Seifert matrix

PEDRO M. GonZÁlez MANCHÓN

Quantum affine algebras, canonical bases, and $q$-deformation of arithmetical functions

HENRY H. KIM and KYU-HWAN LEE

Dirichlet-Ford domains and arithmetic reflection groups

GRANT S. LAKELAND

Formal equivalence of Poisson structures around Poisson submanifolds

IOAN MĂRCUT,

A regularity theorem for graphic spacelike mean curvature flows

BENJAMIN STUART THORPE

Analogues of level- $N$ Eisenstein series 